Discrete Comput Geom 29:525-560 (2003)

DOI: $10.1007 / \mathrm{s} 00454-003-0781-\mathrm{z}$

\title{
Consequences of Pure Point Diffraction Spectra for Multiset Substitution Systems*
}

\author{
Jeong-Yup Lee, ${ }^{1}$ Robert V. Moody, ${ }^{1}$ and Boris Solomyak ${ }^{2}$ \\ ${ }^{1}$ Department of Mathematical and Statistical Sciences, University of Alberta, \\ Edmonton, Alberta, Canada T6G 2G1 \\ jlee@math.ualberta.ca \\ rmoody@ualberta.ca \\ ${ }^{2}$ Department of Mathematics, University of Washington, \\ Seattle, WA 98195, USA \\ solomyak@math.washington.edu
}

\begin{abstract}
There is a growing body of results in the theory of discrete point sets and tiling systems giving conditions under which such systems are pure point diffractive. Here we look at the opposite direction: what can we infer about a discrete point set or tiling, defined through a primitive substitution system, given that it is pure point diffractive? Our basic objects are Delone multisets and tilings, which are self-replicating under a primitive substitution system of affine mappings with a common expansive map $Q$. Our first result gives a partial answer to a question of Lagarias and Wang: we characterize repetitive substitution Delone multisets that can be represented by substitution tilings using a concept of "legal cluster." This allows us to move freely between both types of objects. Our main result is that for lattice substitution multiset systems (in arbitrary dimensions), being a regular model set is not only sufficient for having pure point spectrum-a known fact—but is also necessary.

This completes a circle of equivalences relating pure point dynamical and diffraction spectra, modular coincidence, and model sets for lattice substitution systems begun by the first two authors of this paper.
\end{abstract}

\section{Introduction}

One of the forces behind the development of the theory of aperiodic order is the unexpected ability of aperiodic structures to display perfect pure point diffraction. Until the mid-1980s, this type of phenomenon had been considered, at least physically, to be

\footnotetext{
* The second author is grateful for the continuing support of an NSERC Operating Grant in this research, and the third author acknowledges support from NSF Grants DMS 9800786 and DMS 0099814.
} 
strictly indicative of crystals. Its discovery in new non-crystallographic metallic solids (now called quasicrystals) and in mathematically aperiodic structures like the Penrose tilings made it clear just how poor our understanding of diffraction had been.

Over the past 15 years a great number of diffractive aperiodic tilings and point sets have been discovered, so that by now there is no shortage of examples. Still, there remains the fundamental and difficult question: given a pure point diffraction pattern, what can we say about where it came from? In this generality we have little hope of answering the question, partly because diffraction is only an average statistical property of the structure creating it and partly because it is well known that in any case the diffraction pattern does not contain enough mathematical information to allow for the solution.

However, if a priori we know something of the structure of the object producing the pattern, then it is possible to make progress. This is the key behind practical algorithms for solving this inverse problem in the field of crystallography. In the case of aperiodic structures the things are, of course, more difficult. However, if we know that the originating structure is a discrete set of points arising, say, by marking the points of a substitution tiling then already we have a good deal of prior information, and in such a situation we can draw useful conclusions.

This paper addresses this type of problem. The basic objects of our study are tilings and certain colored Delone point sets (multisets) in Euclidean space $\mathbb{R}^{d}$, arising from primitive substitution systems (see Section 2 for the definitions). The underlying assumption that we make throughout is that for each $R>0$, the number of configurations, up to translation, of diameter less than $R$ of our tilings or multisets is finite, a condition called finite local complexity. The main problem is to relate the diffractive properties, especially pure point diffraction, with the geometrical properties of our tilings or multisets and the substitution systems that create them. In the case of multisets which form a partition of a lattice in $\mathbb{R}^{d}$ these geometrical properties assume the particularly explicit form of model sets (cut and project sets).

A substitution is a finite collection of affine mappings whose linear part is a common expansive mapping $Q$ on $\mathbb{R}^{d}$ (see Definitions 3.1 and 3.4). Substitutions are familiar in tilings and are the basic method of construction of many of the most famous aperiodic examples. The idea is even simpler for multisets.

Lagarias and Wang [9] have developed a theory for substitution Delone multisets that, under certain conditions, allows one to represent them by substitution tilings in which each tile exactly corresponds to one point in the multisets (such Delone multisets are called representable). Parenthetically we note that this is considerably more subtle than simply performing the Voronoi construction or one of its relatives, which generally does not create a tiling inheriting the underlying substitution. The "inflated" tiles should decompose into unions of tiles exactly. In fact, the Lagarias and Wang tiles may have "fractal" boundaries, as in Example 3.11 below.

A question raised in [9] was to characterize representable tilings. The first new result of the paper (Theorem 3.7) gives a partial answer. We introduce a notion of legal cluster and prove that under the assumption of repetitivity, legality of all clusters is equivalent to representability by a tiling. This result enables us to pass freely between substitution Delone multisets and substitution tilings, and to infer certain properties of substitution Delone multisets from corresponding properties of substitution tilings. For example, we are able to prove that the dynamical systems of primitive substitution Delone multisets for which every cluster is legal are uniquely ergodic (Corollary 4.9). 
A point set is pure point diffractive, or has a "perfect diffraction pattern," if the Fourier transform of its autocorrelation measure is a pure point measure. The reader is referred to [8] or [11] for more on this. In [11] we proved that, under the assumption of unique ergodicity, pure point diffractivity implies that the corresponding dynamical system has a pure discrete dynamical spectrum, the converse of an earlier result by Dworkin [5]. Thus in this paper we are able to restrict ourselves to the framework of the spectra of dynamical systems, which we do.

Section 4 contains Theorem 4.7 and Corollary 4.10 which provide necessary and sufficient conditions for pure pointedness in terms of the asymptotic behavior of the $Q$ iterates of almost-periods of the system. The theorem itself is a generalization from two to arbitrary dimension of a result in [17]. At the present stage of our knowledge we could not prove these conditions directly, without passing to the associated substitution tilings.

Section 5 contains our main results which are an application of the foregoing theory to the case of lattice substitution systems. These are substitution multisets for which the multiset is a partition of a lattice. The objective here is to relate pure pointedness to the theory of model sets (or cut and project sets). We prove that being a regular model set is not only sufficient for having pure point spectrum —a known fact—but is also necessary for certain lattice substitution multiset systems (in arbitrary dimensions). This completes a circle of equivalences begun in [10].

The final result (Theorem 5.12) links modular coincidence, the existence of a model set interpretation, the two notions of pure point spectrum, and the aforementioned condition on almost-periods. The main parts of this that are new to this paper are Theorem 5.6 and the reworking of the theory of lattice substitutions begun in [10] to take account of the more refined definition of modular coincidence required here. Theorem 5.12 provides, in particular, an extension of Dekking's well-known criterion for pure point spectrum in terms of coincidences, generalizing it from its original one-dimensional setting in constant-length alphabetic substitutions [4]. A number of interesting tilings fall into the setting of lattice substitutions, including the Robinson [15], sphinx, and chair tilings [10].

The paper concludes with an Appendix of two subsections. One establishes the uniform patch frequency property of fixed points of primitive tiling substitution systems. This is already known in various forms, but we offer a proof here since it is hard to find a version in the literature that is fitted to our present needs. The uniform patch frequency property is equivalent to the unique ergodicity of the corresponding dynamical system, which is essential to our arguments on pure point diffractivity. The second part of the Appendix proves the necessity in Theorem 4.7, which is a modification of the argument in [17].

\section{Definitions and Notation}

\subsection{Delone Multisets}

A multiset ${ }^{1}$ or $m$-multiset in $\mathbb{R}^{d}$ is a subset $\Lambda=\Lambda_{1} \times \cdots \times \Lambda_{m} \subset \mathbb{R}^{d} \times \cdots \times \mathbb{R}^{d}$ ( $m$ copies) where $\Lambda_{i} \subset \mathbb{R}^{d}$. We also write $\Lambda=\left(\Lambda_{1}, \ldots, \Lambda_{m}\right)=\left(\Lambda_{i}\right)_{i \leq m}$. Recall that a Delone set

\footnotetext{
${ }^{1}$ Caution: in [9], which we occasionally cite below, the word multiset refers to a set with multiplicities.
} 
is a relatively dense and uniformly discrete subset of $\mathbb{R}^{d}$. We say that $\Lambda=\left(\Lambda_{i}\right)_{i \leq m}$ is a Delone multiset in $\mathbb{R}^{d}$ if each $\Lambda_{i}$ is Delone and $\operatorname{supp}(\boldsymbol{\Lambda}):=\bigcup_{i=1}^{m} \Lambda_{i} \subset \mathbb{R}^{d}$ is Delone.

Although $\Lambda$ is a product of sets, it is convenient to think of it as a set with types or colors, $i$ being the color of points in $\Lambda_{i}$. A cluster of $\Lambda$ is, by definition, a family $\mathbf{P}=\left(P_{i}\right)_{i \leq m}$ where $P_{i} \subset \Lambda_{i}$ is finite for all $i \leq m$. Many of the clusters that we consider have the form $A \cap \boldsymbol{\Lambda}:=\left(A \cap \Lambda_{i}\right)_{i \leq m}$, for a bounded set $A \subset \mathbb{R}^{d}$. There is a natural translation $\mathbb{R}^{d}$-action on the set of Delone multisets and their clusters in $\mathbb{R}^{d}$. The translate of a cluster $\mathbf{P}$ by $x \in \mathbb{R}^{d}$ is $x+\mathbf{P}=\left(x+P_{i}\right)_{i \leq m}$. We say that two clusters $\mathbf{P}$ and $\mathbf{P}^{\prime}$ are translationally equivalent if $\mathbf{P}=x+\mathbf{P}^{\prime}$ for some $x \in \mathbb{R}^{d}$. For any two Delone $m$-multisets $\boldsymbol{\Lambda}, \boldsymbol{\Gamma}$ we define $\boldsymbol{\Lambda} \cap \boldsymbol{\Gamma}=\left(\Lambda_{i} \cap \Gamma_{i}\right)_{i \leq m}$ and $\boldsymbol{\Lambda} \Delta \boldsymbol{\Gamma}=\left(\Lambda_{i} \Delta \Gamma_{i}\right)_{i \leq m}$, where $\Lambda_{i} \triangle \Gamma_{i}=\left(\Lambda_{i} \backslash \Gamma_{i}\right) \cup\left(\Gamma_{i} \backslash \Lambda_{i}\right)$. We write $B_{R}(y)$ for the closed ball of radius $R$ centered at $y$ and use also $B_{R}$ for $B_{R}(0)$.

Definition 2.1. A Delone multiset $\Lambda$ has finite local complexity (FLC) if for every $R>0$ there exists a finite set $Y \subset \operatorname{supp}(\boldsymbol{\Lambda})=\bigcup_{i=1}^{m} \Lambda_{i}$ such that

$$
\forall x \in \operatorname{supp}(\boldsymbol{\Lambda}), \quad \exists y \in Y, \quad B_{R}(x) \cap \mathbf{\Lambda}=\left(B_{R}(y) \cap \boldsymbol{\Lambda}\right)+(x-y) .
$$

In plain language, for each radius $R>0$ there are only finitely many translational classes of clusters whose support lies in some ball of radius $R$.

In this paper we usually assume that our Delone multisets have FLC.

Definition 2.2. A Delone multiset $\Lambda$ is repetitive if for any compact set $K \subset \mathbb{R}^{d}$, $\left\{t \in \mathbb{R}^{d}: \boldsymbol{\Lambda} \cap K=(t+\boldsymbol{\Lambda}) \cap K\right\}$ is relatively dense; i.e. there exists $R=R(K)>0$ such that every open ball $B_{R}(y)$ contains at least one element of $\left\{t \in \mathbb{R}^{d}: \boldsymbol{\Lambda} \cap K=(t+\boldsymbol{\Lambda}) \cap K\right\}$.

For a cluster $\mathbf{P}$ and a bounded set $A \subset \mathbb{R}^{d}$ denote

$$
L_{\mathbf{P}}(A)=\sharp\left\{x \in \mathbb{R}^{d}: x+\mathbf{P} \subset A \cap \boldsymbol{\Lambda}\right\},
$$

where $\sharp$ means the cardinality. In plain language, $L_{\mathbf{P}}(A)$ is the number of translates of $\mathbf{P}$ contained in $A$, which is clearly finite. For a bounded set $F \subset \mathbb{R}^{d}$ and $r>0$, let

$$
\begin{aligned}
& F^{+r}:=\left\{x \in \mathbb{R}^{d}: \operatorname{dist}(x, F) \leq r\right\}, \\
& F^{-r}:=\{x \in F: \operatorname{dist}(x, \partial F) \geq r\} \supset F \backslash(\partial F)^{+r} .
\end{aligned}
$$

A van Hove sequence for $\mathbb{R}^{d}$ is a sequence $\mathcal{F}=\left\{F_{n}\right\}_{n \geq 1}$ of bounded measurable subsets of $\mathbb{R}^{d}$ satisfying

$$
\lim _{n \rightarrow \infty} \operatorname{Vol}\left(\left(\partial F_{n}\right)^{+r}\right) / \operatorname{Vol}\left(F_{n}\right)=0 \quad \text { for all } \quad r>0 .
$$

Throughout this paper we deal with concepts that depend on some sort of averaging sequence for their very definition: densities, frequencies, autocorrelation, and diffraction measures. Even when not explicitly mentioned, we always have in mind that these concepts have been defined in terms of some predetermined van Hove sequence $\left\{F_{n}\right\}_{n \geq 1}$. 
Definition 2.3. Let $\left\{F_{n}\right\}_{n \geq 1}$ be a van Hove sequence. The Delone multiset $\Lambda$ has uniform cluster frequencies (UCF) (relative to $\left\{F_{n}\right\}_{n \geq 1}$ ) if for any cluster $\mathbf{P}$ there is the limit

$$
\operatorname{freq}(\mathbf{P}, \boldsymbol{\Lambda})=\lim _{n \rightarrow \infty} \frac{L_{\mathbf{P}}\left(x+F_{n}\right)}{\operatorname{Vol}\left(F_{n}\right)} \geq 0,
$$

uniformly in $x \in \mathbb{R}^{d}$.

For any subset $\Lambda^{\prime} \subset \Lambda$, we define

$$
\operatorname{dens}\left(\boldsymbol{\Lambda}^{\prime}\right):=\lim _{n \rightarrow \infty} \frac{\sharp\left(\boldsymbol{\Lambda}^{\prime} \cap F_{n}\right)}{\operatorname{Vol}\left(F_{n}\right)}
$$

if the limit exists.

Let $\Lambda$ be a Delone multiset and let $X$ be the collection of all Delone multisets each of whose clusters is a translate of a $\boldsymbol{\Lambda}$-cluster. We introduce a metric on Delone multisets in a simple variation of the standard way: for Delone multisets $\Lambda_{1}, \Lambda_{2} \in X$,

$$
d\left(\boldsymbol{\Lambda}_{1}, \boldsymbol{\Lambda}_{2}\right):=\min \left\{\tilde{d}\left(\boldsymbol{\Lambda}_{1}, \boldsymbol{\Lambda}_{2}\right), 2^{-1 / 2}\right\},
$$

where

$$
\begin{aligned}
\tilde{d}\left(\boldsymbol{\Lambda}_{1}, \boldsymbol{\Lambda}_{2}\right)=\inf \left\{\varepsilon>0: \exists x, y \in B_{\varepsilon}(0),\right. \\
\left.B_{1 / \varepsilon}(0) \cap\left(-x+\boldsymbol{\Lambda}_{1}\right)=B_{1 / \varepsilon}(0) \cap\left(-y+\boldsymbol{\Lambda}_{2}\right)\right\} .
\end{aligned}
$$

For a proof of this, see [11].

We define $X_{\Lambda}:=\overline{\left\{-h+\Lambda: h \in \mathbb{R}^{d}\right\}}$ with the metric $d$. In spite of the special role played by 0 in the definition of $d$, any other point of $\mathbb{R}^{d}$ may be used as a reference point, leading to an equivalent metric and more importantly the same topology on $X_{\Lambda}$.

The group $\mathbb{R}^{d}$ acts on $X_{\Lambda}$ by translations which are obviously homeomorphisms, and we get a topological dynamical system $\left(X_{\Lambda}, \mathbb{R}^{d}\right)$.

\subsection{Tilings}

This subsection briefly reviews the basic definitions of tilings and their associated dynamical systems. We begin with a set of types (or colors) $\{1, \ldots, m\}$, which we fix once and for all. A tile in $\mathbb{R}^{d}$ is defined as a pair $T=(A, i)$ where $A=\operatorname{supp}(T)$ (the support of $T)$ is a compact set in $\mathbb{R}^{d}$ which is the closure of its interior, and $i=l(T) \in\{1, \ldots, m\}$ is the type of $T$. We let $g+T=(g+A, i)$ for $g \in \mathbb{R}^{d}$. We say that a set $P$ of tiles is a patch if the number of tiles in $P$ is finite and the tiles of $P$ have mutually disjoint interiors (strictly speaking, we have to say "supports of tiles," but this abuse of language should not lead to confusion). The support of a patch is the union of the supports of the tiles that are in it. Note that the support of a patch need not be connected. The $d i$ ameter of a patch is the diameter of its support. The translate of a patch $P$ by $g \in \mathbb{R}^{d}$ is $g+P:=\{g+T: T \in P\}$. We say that two patches $P_{1}$ and $P_{2}$ are translationally equivalent if $P_{2}=g+P_{1}$ for some $g \in \mathbb{R}^{d}$. A tiling of $\mathbb{R}^{d}$ is a set $\mathcal{T}$ of tiles such that 
$\mathbb{R}^{d}=\bigcup\{\operatorname{supp}(T): T \in \mathcal{T}\}$ and distinct tiles have disjoint interiors. Given a tiling $\mathcal{T}$, finite sets of tiles of $\mathcal{T}$ are called $\mathcal{T}$-patches.

We define FLC, repetitivity, and uniform patch frequencies (UPF), which is the analog of UCF, on tilings in the same way as the corresponding properties on Delone multisets.

We always assume that:

- Any two $\mathcal{T}$-tiles with the same color are translationally equivalent. (Hence there are finitely many $\mathcal{T}$-tiles up to translation.)

- The tiling $\mathcal{T}$ has FLC, that is, for any $R>0$ there are finitely many $\mathcal{T}$-patches of diameter less than $R$ up to translation equivalence.

Let $X_{\mathcal{T}}=\overline{\left\{-g+\mathcal{T}: g \in \mathbb{R}^{d}\right\}}$, where $X_{\mathcal{T}}$ carries a well-known topology, given analogously to (2.2) for $X_{\Lambda}$, relative to which it is compact (equivalent to FLC). We have a natural action of $\mathbb{R}^{d}$ on $X_{\mathcal{T}}$ which makes it a topological dynamical system. The set $\left\{-g+\mathcal{T}: g \in \mathbb{R}^{d}\right\}$ is the orbit of $\mathcal{T}$. A dynamical system is minimal if the orbit of every element of $X_{\mathcal{T}}$ is dense in $X_{\mathcal{T}}$. The minimality of dynamical system $\left(X_{\mathcal{T}}, \mathbb{R}^{d}\right)$ is equivalent to the repetitivity of $\mathcal{T}$ (see [6]).

Definition 2.4. Let $P$ be a patch of $\mathcal{T}$ or some translate of $\mathcal{T}$, and let $W \subset \mathbb{R}^{d}$ be a Borel set. Define the cylinder set $X_{P, W}$ by

$$
X_{P, W}:=\left\{\mathcal{S} \in X_{\mathcal{T}}:-g+P \text { is an } \mathcal{S} \text {-patch for some } g \in W\right\} .
$$

\section{Substitution Systems}

\subsection{Tile-Substitutions}

We say that a linear map $Q: \mathbb{R}^{d} \rightarrow \mathbb{R}^{d}$ is expansive if there is a $c>1$ with

$$
d(Q x, Q y) \geq c \cdot d(x, y)
$$

for all $x, y \in \mathbb{R}^{d}$ and some metric $d$ on $\mathbb{R}^{d}$ compatible with the standard topology. This is equivalent to saying that all the eigenvalues of $Q$ lie outside the closed unit disk in $\mathbb{C}$.

Definition 3.1. Let $\mathcal{A}=\left\{T_{1}, \ldots, T_{m}\right\}$ be a finite set of tiles in $\mathbb{R}^{d}$ such that $T_{i}=\left(A_{i}, i\right)$; we call them prototiles. Denote by $\mathcal{P}_{\mathcal{A}}$ the set of patches made of tiles each of which is a translate of one of the $T_{i}$ 's. We say that $\omega: \mathcal{A} \rightarrow \mathcal{P}_{\mathcal{A}}$ is a tile-substitution (or simply substitution) with expansive map $Q$ if there exist finite sets $\mathcal{D}_{i j} \subset \mathbb{R}^{d}$ for $i, j \leq m$, such that

$$
\omega\left(T_{j}\right)=\left\{u+T_{i}: u \in \mathcal{D}_{i j}, i=1, \ldots, m\right\} \quad \text { for } \quad j \leq m,
$$

with

$$
Q\left(A_{j}\right)=\bigcup_{i=1}^{m}\left(\mathcal{D}_{i j}+A_{i}\right) .
$$

Here all sets in the right-hand side must have disjoint interiors; it is possible for some of the $\mathcal{D}_{i j}$ to be empty. 
The substitution (3.2) is extended to all translates of prototiles by $\omega\left(x+T_{j}\right)=$ $Q x+\omega\left(T_{j}\right)$, and to patches and tilings by $\omega(P)=\bigcup\{\omega(T): T \in P\}$. The substitution $\omega$ can be iterated, producing larger and larger patches $\omega^{k}\left(T_{j}\right)$. To the substitution $\omega$ we associate its $m \times m$ substitution matrix $S$, with $S_{i j}:=\sharp\left(\mathcal{D}_{i j}\right)$. The substitution $\omega$ is called primitive if the substitution matrix $S$ is primitive, i.e. there is an $l>0$ for which $S^{l}$ has no zero entries.

Definition 3.2. A patch will be called legal if it is a translate of a subpatch of $\omega^{k}\left(T_{i}\right)$ for some $i \leq m$ and $k \geq 1$. A tiling $\mathcal{T}$ with FLC is said to be self-affine with the prototile set $\mathcal{A}$, expansive map $Q$, and primitive substitution $\omega$, if every $\mathcal{T}$-patch is legal.

The set of self-affine tilings associated with $(\mathcal{A}, \omega)$ will be denoted by $X_{\mathcal{A}, \omega}$. A tiling $\mathcal{T}$ is called a fixed point of the substitution $\omega$ if $\omega(\mathcal{T})=\mathcal{T}$. It turns out that such a tiling need not be repetitive, even though the substitution is primitive, see [18]. In that case one can show that $X_{\mathcal{T}} \supset X_{\mathcal{A}, \omega}$ but this inclusion is proper. It is well known (and easy to see) that one can always find a periodic point for $\omega$ in the space $X_{\mathcal{A}, \omega}$, i.e. there is $\mathcal{T} \in X_{\mathcal{A}, \omega}$ such that $\omega^{N}(\mathcal{T})=\mathcal{T}$ for some $N \geq 1$. In this case we can always use $\omega^{N}$ in place of $\omega$ to obtain a tiling which is a fixed point.

Lemma 3.3. Let $\mathcal{T}$ be a fixed point of a primitive substitution $\omega$ with expansive map $Q$ and prototiles $\mathcal{A}$. Then $\mathcal{T}$ is repetitive if and only if every $\mathcal{T}$-patch is legal, i.e. $\mathcal{T} \in X_{\mathcal{A}, \omega}$.

Proof. Suppose $\mathcal{T}$ is repetitive. Then for any patch $P$ of $\mathcal{T}$ there exists $R>0$ such that every open ball $B_{R}(y)$ contains a patch translationally equivalent to $P$. Since each tile of $\mathcal{T}$ has non-empty interior and $\omega$ is with expansive map $Q$, there is $M \geq 1$ such that for any $i \leq m, Q^{M}\left(A_{i}\right)$ contains $B_{R}(y)$ for some $y \in \mathbb{R}^{d}$. This means that $\omega^{M}\left(T_{i}\right)$ contains a patch translationally equivalent to $P$ for any $i \leq m$. Thus every $\mathcal{T}$-patch is legal.

Conversely, suppose every $\mathcal{T}$-patch is legal. Then for every $\mathcal{T}$-patch $P$ there is $K \geq 1$ such that for any $i \leq m, P$ is a translate of a subpatch of $\omega^{K}\left(T_{i}\right)$ by the primitivity of $\omega$. Choose $r>\max \left\{\operatorname{diam}\left(T_{i}\right): i \leq m\right\}$. Every open ball $B_{r}(y)$ contains at least one tile. So every open ball $B_{\|Q\|^{K} r}(y)$, where $\|Q\|$ is the operator norm, contains at least one supertile $\omega^{K}(T)$, which contains a translate of $P$. Therefore $\mathcal{T}$ is repetitive.

\subsection{From Substitution Delone Multisets to Tilings}

We now link the theory of multisets and tilings through the notion of representable Delone multisets. The concept was introduced by Lagarias and Wang [9], under the name of self-replicating Delone set families.

Definition 3.4. $\boldsymbol{\Lambda}=\left(\Lambda_{i}\right)_{i \leq m}$ is called a substitution Delone multiset if $\boldsymbol{\Lambda}$ is a Delone multiset and there exist an expansive map $Q: \mathbb{R}^{d} \rightarrow \mathbb{R}^{d}$ and finite sets $\mathcal{D}_{i j}$ for $i, j \leq m$ such that

$$
\Lambda_{i}=\bigcup_{j=1}^{m}\left(Q \Lambda_{j}+\mathcal{D}_{i j}\right), \quad i \leq m,
$$

where the unions on the right-hand side are disjoint. 
We say that the substitution Delone multiset is primitive if the corresponding substitution matrix $S$, with $S_{i j}=\sharp\left(\mathcal{D}_{i j}\right)$, is primitive.

Let $Y$ be a non-empty set in $\mathbb{R}^{d}$. For $m \in \mathbb{Z}_{+}$, an $m \times m$ matrix function system (MFS) on $Y$ is an $m \times m$ matrix $\Phi=\left(\Phi_{i j}\right)$, where each $\Phi_{i j}$ is a finite set (possibly empty) of mappings $Y$ to $Y$.

Any MFS $\Phi$ on $Y$ induces a mapping on Delone multiset $\Lambda=\left(\Lambda_{i}\right)_{i \leq m}$, where $\Lambda_{i} \subset Y, i \leq m$, by

$$
\Phi\left[\begin{array}{c}
\Lambda_{1} \\
\vdots \\
\Lambda_{m}
\end{array}\right]=\left[\begin{array}{c}
\bigcup_{j \leq m} \bigcup_{f \in \Phi_{1 j}} f\left(\Lambda_{j}\right) \\
\vdots \\
\bigcup_{j \leq m} \bigcup_{f \in \Phi_{m j}} f\left(\Lambda_{j}\right)
\end{array}\right],
$$

which we call the substitution determined by $\Phi$. We often write $\Phi_{i j}\left(\Gamma_{j}\right)$ for $\bigcup_{f \in \Phi_{i j}} f\left(\Gamma_{j}\right)$, $\Phi\left(\Gamma_{j}\right)$ for $\left(\Phi_{i j}\left(\Gamma_{j}\right)\right)_{i \leq m}$, and $\Phi(\boldsymbol{\Gamma})$ for $\left(\bigcup_{j \leq m} \Phi_{i j}\left(\Gamma_{j}\right)\right)_{i \leq m}$ for any subset $\boldsymbol{\Gamma}=\left(\Gamma_{j}\right)_{j \leq m} \subset$ $\Lambda$. In particular, we often write $\Phi_{i j}(x)$ for $\Phi_{i j}(\{x\})$, where $x \in \Lambda_{j}$. We associate to $\Phi$ its substitution matrix $S(\Phi)$, with $(S(\Phi))_{i j}=\sharp\left(\Phi_{i j}\right)$.

Let $\Phi, \Psi$ be $m \times m$ MFSs on $Y$. Then we can compose them:

$$
\Psi \circ \Phi=\left((\Psi \circ \Phi)_{i j}\right)
$$

where

$$
(\Psi \circ \Phi)_{i j}=\bigcup_{k=1}^{m} \Psi_{i k} \circ \Phi_{k j} \quad \text { and } \quad \Psi_{i k} \circ \Phi_{k j}:=\left\{\begin{array}{l}
\left\{g \circ f: g \in \Psi_{i k}, f \in \Phi_{k j}\right\}, \\
\emptyset \text { if } \Psi_{i k}=\emptyset \text { or } \Phi_{k j}=\emptyset .
\end{array}\right.
$$

Evidently, $S(\Psi \circ \Phi) \leq S(\Psi) S(\Phi)$.

For any given substitution Delone multiset $\Lambda=\left(\Lambda_{i}\right)_{i \leq m}$, we can always find the corresponding MFS $\Phi$ such that $\Phi(\boldsymbol{\Lambda})=\boldsymbol{\Lambda}$. Indeed, by Definition 3.4 we can take $\Phi_{i j}=\left\{f: f: x \mapsto Q x+a, a \in \mathcal{D}_{i j}\right\}$. So $\Phi\left(\Lambda_{j}\right)=\left(Q \Lambda_{j}+\mathcal{D}_{i j}\right)_{i \leq m}, j \leq m$.

Lemma 3.5 [9, Theorem 2.3]. If $\boldsymbol{\Lambda}$ is a primitive substitution Delone multiset with expansive map $Q$, then the Perron-Frobenius $(P F)$ eigenvalue of its substitution matrix $S$ equals $|\operatorname{det}(Q)|$.

For each primitive substitution Delone multiset there is an adjoint system of equations

$$
Q A_{j}=\bigcup_{i=1}^{m}\left(\mathcal{D}_{i j}+A_{i}\right), \quad j \leq m .
$$

From Hutchinson's Theory (or, rather, its generalization to the "graph-directed" setting), it follows that (3.6) always has a unique solution for which $\left(A_{i}\right)_{i \leq m}$ is a family of non-empty compact sets of $\mathbb{R}^{d}$ (see for example Proposition 1.3 of [1]). It is proved in Theorems 2.4 and 5.5 of [9] that if $\Lambda$ is a primitive substitution Delone multiset, then all the sets $A_{i}$ from (3.6) have non-empty interior and, moreover, each $A_{i}$ is the closure of its interior.

Definition 3.6. A Delone multiset $\Lambda=\left(\Lambda_{i}\right)_{i \leq m}$ is called representable (by tiles) if there exist tiles $T_{i}=\left(A_{i}, i\right), i \leq m$, so that

$$
\left\{x+T_{i}: x \in \Lambda_{i}, i \leq m\right\} \quad \text { is a tiling of } \mathbb{R}^{d},
$$


that is, $\mathbb{R}^{d}=\bigcup_{i \leq m} \bigcup_{x \in \Lambda_{i}}\left(x+A_{i}\right)$, and the sets in this union have disjoint interiors. In the case that $\Lambda$ is a primitive substitution Delone multiset we will understand the term representable to mean relative to the tiles $T_{i}=\left(A_{i}, i\right), i \leq m$, arising from the solution to the adjoint system (3.6).

A cluster will be called legal if it is a translate of a subcluster of $\Phi^{k}\left(x_{j}\right)$ for some $x_{j} \in \Lambda_{j}, j \leq m$, and some $k \in \mathbb{Z}_{+}$.

Lagarias and Wang have a condition, namely the existence of a fundamental cycle of period 1, which ensures representability. Legality generalizes this and in fact our Theorem 3.7 is based on Theorem 7.1 of [9].

In the same paper [9, Lemma 3.2] it is shown that if $\boldsymbol{\Lambda}$ is a substitution Delone multiset, then there is a finite multiset (cluster) $\mathbf{P} \subset \boldsymbol{\Lambda}$ for which $\Phi^{n-1}(\mathbf{P}) \subset \Phi^{n}(\mathbf{P})$ for $n \geq 1$ and $\boldsymbol{\Lambda}=\lim _{n \rightarrow \infty} \Phi^{n}(\mathbf{P})$. We call such a multiset $\mathbf{P}$ a generating multiset.

Theorem 3.7. Let $\boldsymbol{\Lambda}$ be a primitive substitution Delone multiset such that every $\boldsymbol{\Lambda}$ cluster is legal. Then $\Lambda$ is representable.

Proof. Let $\Phi$ be the MFS satisfying $\Phi(\boldsymbol{\Lambda})=\boldsymbol{\Lambda}$ and suppose $\mathcal{A}=\left\{T_{1}, \ldots, T_{m}\right\}$ arises from the solution to the adjoint system (3.6). So for any $M \in \mathbb{Z}_{+}$,

$$
Q^{M}\left(A_{j}\right)=\bigcup_{i=1}^{m}\left(\left(\mathcal{D}^{M}\right)_{i j}+A_{i}\right), \quad j \leq m
$$

where $A_{i}=\operatorname{supp}\left(T_{i}\right)$ and

$$
\left(\mathcal{D}^{M}\right)_{i j}=\bigcup_{k_{1}, k_{2}, \ldots, k_{(M-1)} \leq m}\left(\mathcal{D}_{i k_{1}}+Q \mathcal{D}_{k_{1} k_{2}}+\cdots+Q^{M-1} \mathcal{D}_{k_{(M-1)} j}\right) .
$$

On the other hand, for any $M \in \mathbb{Z}_{+}$and $i \leq m$,

$$
\left(\Phi^{M}\right)_{i j}\left(x_{j}\right)=Q^{M} x_{j}+\left(\mathcal{D}^{M}\right)_{i j} \quad \text { for any } \quad x_{j} \in \Lambda_{j}, \quad j \leq m .
$$

So putting (3.8) and (3.9) together, we get

$$
\begin{aligned}
Q^{M}\left(x_{j}+A_{j}\right) & =\bigcup_{i=1}^{m}\left(Q^{M} x_{j}+\left(\mathcal{D}^{M}\right)_{i j}+A_{i}\right) \\
& =\bigcup_{i=1}^{m}\left(\left(\Phi^{M}\right)_{i j}\left(x_{j}\right)+A_{i}\right) \\
& =\bigcup_{i=1}^{m} \bigcup_{y \in\left(\Phi^{M}\right)_{i j}\left(x_{j}\right)}\left(y+A_{i}\right) \quad \text { for any } \quad x_{j} \in \Lambda_{j}, \quad j \leq m .
\end{aligned}
$$

From Theorems 2.4 and 5.5 of [9], $\mu\left(A_{j}\right)>0$ and $A_{j}$ is the closure of its interior for any $j \leq m$. Let $\tilde{\mu}:=\left(\mu\left(A_{1}\right), \ldots, \mu\left(A_{m}\right)\right)>0$. Taking measures in (3.10),

$$
|\operatorname{det} Q|^{M} \tilde{\mu} \leq \tilde{\mu} S\left(\Phi^{M}\right) \leq \tilde{\mu} S(\Phi)^{M},
$$


where $S(\Phi)$ is the substitution matrix of $\Phi$. From Theorem 2.3 of [9], we know that $|\operatorname{det} Q|=\mathrm{PF}$ eigenvalue of $S(\Phi)$. So we can derive

$$
|\operatorname{det} Q|^{M} \tilde{\mu}=\tilde{\mu} S(\Phi)^{M}
$$

from (3.11), see Lemma 1 of [10]. Thus for any $x_{j} \in \Lambda_{j}, j \leq m$,

$$
\begin{aligned}
\mu\left(Q^{M}\left(x_{j}+A_{j}\right)\right) & =\mu\left(\bigcup_{i=1}^{m}\left(\left(\Phi^{M}\right)_{i j}\left(x_{j}\right)+A_{i}\right)\right) \\
& =\sum_{i=1}^{m}\left(S\left(\Phi^{M}\right)\right)_{i j} \mu\left(A_{i}\right),
\end{aligned}
$$

and this shows that the unions on the right-hand side of (3.10) are measurewise disjoint. So we get a (tile)-substitution $\omega: \mathcal{A} \rightarrow \mathcal{P}_{\mathcal{A}}$ associated with $\mathcal{A}$ and $\boldsymbol{\Lambda}$.

Next, let $\mathbf{P}$ be a generating multiset. From the assumption that every cluster in $\boldsymbol{\Lambda}$ is legal, there is $a_{l} \in \Lambda_{l}$ for some $l \leq m$ such that $z+\mathbf{P}=\left(z+P_{j}\right)_{j \leq m} \subset\left(\left(\Phi^{K}\right)_{j l}\left(a_{l}\right)\right)_{j \leq m}$ for some $K \in \mathbb{Z}_{+}$and some $z \in \mathbb{R}^{d}$. So

$$
\left\{x+T_{j}: x \in\left(\Phi^{K}\right)_{j l}\left(a_{l}\right), j \leq m\right\} \supset\left\{x+T_{j}: x \in\left(z+P_{j}\right), j \leq m\right\} .
$$

By (3.12), all the tiles in the left-hand side of (3.13) are measurewise disjoint. So then are the tiles in the right-hand side of (3.13). This implies that all the tiles in the set $\left\{p_{j}+T_{j}: p_{j} \in P_{j}, j \leq m\right\}$ are measurewise disjoint. Thus for any $n \in \mathbb{Z}_{+}$, all the supertiles in the set $\left\{\omega^{n}\left(p_{j}+T_{j}\right): p_{j} \in P_{j}, j \leq m\right\}$ are measurewise disjoint. Noting that

$$
\omega^{n}\left(p_{j}+T_{j}\right)=\left\{x+T_{i}: x \in\left(\Phi^{n}\right)_{i j}\left(p_{j}\right), i \leq m\right\} \quad \text { for each } \quad p_{j} \in P_{j}, \quad j \leq m,
$$

we get that

$$
\Phi^{n}(\mathbf{P})+\mathcal{A}:=\left\{x+T_{i}: x \in\left(\Phi^{n}\right)_{i j}\left(p_{j}\right), p_{j} \in P_{j}, i, j \leq m\right\}
$$

also consists of tiles which are measurewise disjoint. Since $\Phi^{n-1}(\mathbf{P}) \subset \Phi^{n}(\mathbf{P})$ for $n \geq 1$ and $\boldsymbol{\Lambda}=\lim _{n \rightarrow \infty} \Phi^{n}(\mathbf{P}), \boldsymbol{\Lambda}+\mathcal{A}:=\left\{x_{j}+T_{j}: x_{j} \in \Lambda_{j}, j \leq m\right\}$ consists of tiles which are measurewise disjoint. Thus distinct tiles in $\Lambda+\mathcal{A}$ have disjoint interiors.

Now we need prove that $\Lambda+\mathcal{A}$ is a tiling. We define $\operatorname{supp}(\boldsymbol{\Lambda}+\mathcal{A}):=\bigcup\left\{x_{j}+A_{j}: x_{j} \in\right.$ $\left.\Lambda_{j}, j \leq m\right\}$. Then

$$
\begin{aligned}
Q(\operatorname{supp}(\Lambda+\mathcal{A})) & =\bigcup\left\{Q x_{j}+Q A_{j}: x_{j} \in \Lambda_{j}, j \leq m\right\} \\
& =\bigcup\left\{Q x_{j}+\mathcal{D}_{i j}+A_{i}: x_{j} \in \Lambda_{j}, i, j \leq m\right\} \\
& =\bigcup\left\{\Phi_{i j}\left(x_{j}\right)+A_{i}: x_{j} \in \Lambda_{j}, i, j \leq m\right\} \\
& =\bigcup\left\{x_{i}+A_{i}: x_{i} \in \Lambda_{i}, i \leq m\right\} \\
& =\operatorname{supp}(\boldsymbol{\Lambda}+\mathcal{A}) .
\end{aligned}
$$


Suppose $\mathbb{R}^{d} \backslash \operatorname{supp}(\boldsymbol{\Lambda}+\mathcal{A}) \neq \emptyset$. Then there is $z \in \mathbb{R}^{d} \backslash \operatorname{supp}(\boldsymbol{\Lambda}+\mathcal{A})$ and a ball $B_{r}(z)$ with radius $r$ centered at $z$ such that $B_{r}(z) \subset \mathbb{R}^{d} \backslash \operatorname{supp}(\boldsymbol{\Lambda}+\mathcal{A})$, since $\operatorname{supp}(\boldsymbol{\Lambda}+\mathcal{A})$ is a closed set. So for any $N \in \mathbb{Z}_{+}$,

$$
\begin{aligned}
Q^{N}\left(B_{r}(z)\right) \cap \operatorname{supp}(\boldsymbol{\Lambda}+\mathcal{A}) & =Q^{N}\left(B_{r}(z)\right) \cap Q^{N}(\operatorname{supp}(\boldsymbol{\Lambda}+\mathcal{A})) \\
& =Q^{N}\left(B_{r}(z) \cap \operatorname{supp}(\boldsymbol{\Lambda}+\mathcal{A})\right)=\emptyset .
\end{aligned}
$$

However, this is a contradiction, since $\Lambda$ is Delone. Therefore $\Lambda+\mathcal{A}$ is a tiling and so $\Lambda$ is representable.

Corollary 3.8. Let $\Lambda$ be a repetitive primitive substitution Delone multiset. Then every $\boldsymbol{\Lambda}$-cluster is legal if and only if $\boldsymbol{\Lambda}$ is representable.

Proof. We only need to prove the sufficiency direction. Suppose $\boldsymbol{\Lambda}$ is representable. Then we have a tiling $\Lambda+\mathcal{A}=\left\{x_{i}+T_{i}: x_{i} \in \Lambda_{i}, i \leq m\right\}$ with a unique solution $\mathcal{A}=\left\{T_{1}, \ldots, T_{m}\right\}$ of the adjoint system of equations such that $\omega(\boldsymbol{\Lambda}+\mathcal{A})=\boldsymbol{\Lambda}+\mathcal{A}$, where $\omega: \mathcal{A} \rightarrow \mathcal{P}_{\mathcal{A}}$ is a (tile)-substitution. So $\boldsymbol{\Lambda}+\mathcal{A}$ is a fixed point of a primitive substitution $\omega$ with expansive map $Q$. By Lemma 3.3, every $(\boldsymbol{\Lambda}+\mathcal{A})$-patch is legal, since $\Lambda+\mathcal{A}$ is repetitive. Recall that for any $M \in \mathbb{Z}_{+}$,

$$
\omega^{M}\left(x_{j}+T_{j}\right)=\left\{x+T_{i}: x \in\left(\Phi^{M}\right)_{i j}\left(x_{j}\right), i \leq m\right\} \quad \text { for any } \quad x_{j} \in \Lambda_{j}, \quad j \leq m,
$$

where $\Phi$ is the MFS satisfying $\Phi(\boldsymbol{\Lambda})=\boldsymbol{\Lambda}$. So the legality in the tiling $\boldsymbol{\Lambda}+\mathcal{A}$ shows the legality in $\boldsymbol{\Lambda}$. Therefore every $\boldsymbol{\Lambda}$-cluster is legal.

Remark 3.9. Note that in order to check that every $\boldsymbol{\Lambda}$-cluster is legal, we only need to see if some cluster that contains a finite generating multiset for $\boldsymbol{\Lambda}$ is legal.

Any tiling $\mathcal{T}$ can be converted into a Delone multiset by simply choosing a point $x_{(A, i)}$ for each tile $(A, i)$ so that the chosen points for tiles of the same type are in the same relative position in the tile: $x_{(g+A, i)}=g+x_{(A, i)}$. We define $\Lambda_{i}:=\left\{x_{(A, i)}:(A, i) \in \mathcal{T}\right\}$ and $\boldsymbol{\Lambda}:=\left(\Lambda_{i}\right)_{i \leq m}$. Clearly $\mathcal{T}$ can be reconstructed from $\boldsymbol{\Lambda}$ given the information about how the points lie in their respective tiles. This bijection establishes a topological conjugacy of $\left(X_{\boldsymbol{\Lambda}}, \mathbb{R}^{d}\right)$ and $\left(X_{\mathcal{T}}, \mathbb{R}^{d}\right)$. Concepts and theorems can clearly be interpreted in either language (FLC, UCF, unique ergodicity, pure point dynamical spectrum, etc.).

In the case that $\Lambda$ is a representable primitive substitution Delone multiset, we make use of the following bijection. We consider $T_{i}=\left(A_{i}, i\right), i \leq m$, as prototiles, where the $A_{i}$ 's are defined by (3.6). Let $\mathcal{T}=\mathcal{T}(\boldsymbol{\Lambda})$ be the tiling in (3.7), with the colors added, that is, $\mathcal{T}=\left\{x_{i}+T_{i}: x_{i} \in \Lambda_{i}, i \leq m\right\}$, and let $\mathcal{A}=\left\{T_{1}, \ldots, T_{m}\right\}$. By (3.6) and the definition of a representable primitive substitution Delone multiset, we have a tile-substitution $\omega: \mathcal{A} \rightarrow \mathcal{P}_{\mathcal{A}}$.

Lemma 3.10. The dynamical systems $\left(X_{\Lambda}, \mathbb{R}^{d}\right)$ and $\left(X_{\mathcal{T}}, \mathbb{R}^{d}\right)$ are topologically conjugate. 


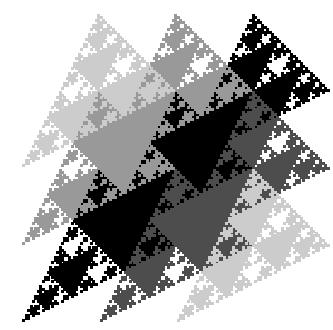

Fig. 1. Gasket tiling.

Proof. For any Delone multiset $\boldsymbol{\Gamma}=\left(\Gamma_{i}\right)_{i \leq m} \in X_{\boldsymbol{\Lambda}}$ we let $\varphi(\boldsymbol{\Gamma})=\left\{x_{i}+T_{i}: x_{i} \in\right.$ $\left.\Gamma_{i}, i \leq m\right\}$. It is straightforward to check that $\varphi(\boldsymbol{\Gamma}) \in X_{\mathcal{T}}$ and $\varphi$ is a homeomorphism commuting with the translation action.

Example 3.11 (A substitution Delone multiset in $\mathbb{R}^{2}$ with gasket tiles). Consider the substitution on $\mathbb{R}^{2}$ with the following MFS $\Phi$ :

$$
\Phi=\left(\begin{array}{cccc}
\left\{f_{1}, f_{4}\right\} & \left\{f_{1}\right\} & \left\{f_{1}\right\} & \emptyset \\
\left\{f_{2}\right\} & \left\{f_{2}, f_{3}\right\} & \emptyset & \left\{f_{2}\right\} \\
\left\{f_{3}\right\} & \emptyset & \left\{f_{2}, f_{3}\right\} & \left\{f_{3}\right\} \\
\emptyset & \left\{f_{4}\right\} & \left\{f_{4}\right\} & \left\{f_{1}, f_{4}\right\}
\end{array}\right),
$$

where $f_{1}(x)=2 x, f_{2}(x)=2 x+(1,0), f_{3}(x)=2 x+(0,1)$, and $f_{4}(x)=2 x+(-1,-1)$.

The Delone multiset $\boldsymbol{\Lambda}=\left(\Lambda_{1}, \Lambda_{2}, \Lambda_{3}, \Lambda_{4}\right)$ generated from $(\{(0,0),(1,1)\},\{(0$, $-1)\},\{(-1,0)\}, \emptyset)$ is fixed under $\Phi$. We observe that $\bigcup_{i \leq 4} \Lambda_{i}=\mathbb{Z}^{2}$. The generating multiset $(\{(0,0),(1,1)\},\{(0,-1)\},\{(-1,0)\}, \emptyset)$ is legal, indicating representability. The solution from the adjoint system consists of four copies of the gasket tile $(T)$ [19]. Thus $\left\{x+T: x \in \Lambda_{i}, i \leq 4\right\}$ is a tiling of $\mathbb{R}^{2}$ by gaskets pinned down on the standard lattice $\mathbb{Z}^{2}$, see Fig. 1. Note that the tiles are triangular gaskets in four colors, indicated by three shades of grey and black. The largest solid triangles of one color (e.g. black) are caused by the meshing of two gaskets of the same color.

Example 3.12 (A substitution Delone multiset for which not every cluster is legal). Consider the MFS $\Phi$ :

$$
\Phi=\left(\begin{array}{cc}
\{5 x, 5 x+2,5 x+4\} & \{5 x+1,5 x+3\} \\
\{5 x+1,5 x+3\} & \{5 x, 5 x+2,5 x+4\}
\end{array}\right)
$$

which generates the bi-infinite sequence shown below with the $a$ and $b$ point sets starting from the generating set $\left(\{0\},\left\{-\frac{1}{2},-1\right\}\right)$. This leads to the Delone multiset $\Lambda=\left(\Lambda_{a}, \Lambda_{b}\right)$ which is fixed under $\Phi$.

\begin{tabular}{lllllllllllllllll}
$\cdots$ & -3 & $-\frac{5}{2}$ & -2 & $-\frac{3}{2}$ & -1 & $-\frac{1}{2}$ & 0 & $\frac{1}{2}$ & 1 & $\frac{3}{2}$ & 2 & $\frac{5}{2}$ & 3 & $\frac{7}{2}$ & 4 & $\cdots$ \\
\hline$\cdots$ & $b$ & $b$ & $a$ & $a$ & $b$ & $b$ & $a$ & $a$ & $b$ & $b$ & $a$ & $a$ & $b$ & $b$ & $a$ & $\cdots$
\end{tabular}

Note that the generating set $\left(\{0\},\left\{-\frac{1}{2},-1\right\}\right)$ is not legal, even though $\boldsymbol{\Lambda}$ is periodic and so repetitive. Thus $\Lambda$ is not representable by tiles arising from the solution to the 
adjoint system. In fact the solution to the adjoint system is $\mathcal{A}=\{[0,1],[0,1]\}$ so that $\Lambda+\mathcal{A}$ double tiles the line.

\section{Pure Point Spectrum}

\subsection{Pure-Pointedness for Tilings}

This subsection is largely based on [17]. The main new feature is that here the dimension $d$ is arbitrary, while [17] was mostly focused on the case $d=2$.

Recall that a topological dynamical system is uniquely ergodic if there is a unique invariant Borel probability measure.

Theorem 4.1. If $\mathcal{T}$ is a fixed point of a primitive substitution with $F L C,{ }^{2}$ then $\mathcal{T}$ has uniform patch frequencies $(U P F)$, and the tiling dynamical system $\left(X_{\mathcal{T}}, \mathbb{R}^{d}\right)$ is uniquely ergodic.

This is already known in various forms but we offer a proof in the Appendix since it is hard to find a version in the literature that is fitted to our present needs. More precisely, in Lemma A.6 we establish the existence of UPF. The fact that UPF implies unique ergodicity is proved in Theorems 2.6 and 2.7 of [11] in the setting of arbitrary Delone multisets with FLC.

The theorem shows that the dynamical system $\left(X_{\mathcal{T}}, \mathbb{R}^{d}\right)$ is ergodic with respect to the unique invariant probability measure on $X_{\mathcal{T}}$, which we denote by $\mu$. We will also need the following result, proved in Corollary 2.8 of [11]. Let $\eta(\mathcal{T})>0$ be chosen such that every tile support contains a ball of diameter $\eta(\mathcal{T})$.

Corollary 4.2. Let $\mathcal{T}$ be a fixed point of a primitive substitution with FLC. Then for any $\mathcal{T}$-patch $P$ and any Borel set $V$ with $\operatorname{diam}(V)<\eta(\mathcal{T})$, we have

$$
\mu\left(X_{P, V}\right)=\operatorname{Vol}(V) \cdot \operatorname{freq}(P, \mathcal{T}) .
$$

We consider the associated group of unitary operators $\left\{U_{g}\right\}_{g \in \mathbb{R}^{d}}$ on $L^{2}\left(X_{\mathcal{T}}, \mu\right)$ :

$$
U_{g} f(\mathcal{S})=f(-g+\mathcal{S}) .
$$

A vector $\alpha=\left(\alpha_{1}, \ldots, \alpha_{d}\right) \in \mathbb{R}^{d}$ is said to be an eigenvalue for the $\mathbb{R}^{d}$-action if there exists an eigenfunction $f \in L^{2}\left(X_{\mathcal{T}}, \mu\right)$, that is, $f \not \equiv 0$ and

$$
U_{g} f(\mathcal{S})=e^{2 \pi i g \cdot \alpha} f(\mathcal{S}) \quad \text { for all } \quad g \in \mathbb{R}^{d} .
$$

The dynamical system $\left(X_{\mathcal{T}}, \mu, \mathbb{R}^{d}\right)$ is said to have pure discrete (or pure point) spectrum if the linear span of the eigenfunctions is dense in $L^{2}\left(X_{\mathcal{T}}\right)$.

Let $\Xi(\mathcal{T})$ be the set of translation vectors between $\mathcal{T}$-tiles of the same type:

$$
\Xi(\mathcal{T})=\left\{x \in \mathbb{R}^{d}: \exists T, T^{\prime} \in \mathcal{T}, T^{\prime}=x+T, i\left(T^{\prime}\right)=i(T)\right\} .
$$

\footnotetext{
${ }^{2}$ Recently Danzer [3] has given an example of a primitive substitution tiling which does not satisfy FLC.
} 
Since $\mathcal{T}$ has the inflation symmetry with the expansive map $Q$, we have that $Q \Xi(\mathcal{T}) \subset$ $\Xi(\mathcal{T})$. Note also that $\Xi(\mathcal{T})=-\Xi(\mathcal{T})$. If $\mathcal{T}=\mathcal{T}(\Lambda)$ is a tiling for a representable Delone multiset $\Lambda$, then $\Xi(\mathcal{T})=\bigcup_{i=1}^{m}\left(\Lambda_{i}-\Lambda_{i}\right)$, see Section 3.2. The following is proved in Section 4 of [17].

Theorem 4.3. Suppose that $\mathcal{T}$ is a repetitive fixed point of a primitive substitution with expansive map $Q$ and $\mathcal{T}$ has FLC. If $\alpha \in \mathbb{R}^{d}$ is an eigenvalue for $\left(X_{\mathcal{T}}, \mu, \mathbb{R}^{d}\right)$, then for any $x \in \Xi(\mathcal{T})$ we have

$$
\lim _{n \rightarrow \infty} e^{2 \pi i\left(Q^{n} x\right) \cdot \alpha}=1
$$

This theorem yields necessary conditions on the expansive map $Q$ for the dynamical system to have non-trivial eigenfunctions. The simplest is that if $Q$ is a diagonal matrix with diagonal entries $\lambda>1$, then $\lambda$ has to be a Pisot number. This follows from the algebraicity of $\lambda$ (easy to see) and the classical theorem of Pisot. Other conditions can be found in [17].

For $x \in \Xi(\mathcal{T})$ consider the infinite subset

$$
D_{x}:=\mathcal{T} \cap(x+\mathcal{T}) .
$$

It is non-empty by (4.1), and $\operatorname{supp}\left(D_{x}\right)$ is relatively dense by repetitivity. Observe that $D_{x}$ has a well-defined density given by

$$
\begin{aligned}
\operatorname{dens}\left(D_{x}\right) & =\lim _{n \rightarrow \infty} \frac{\operatorname{Vol}\left(D_{x} \cap F_{n}\right)}{\operatorname{Vol}\left(F_{n}\right)} \\
& =\sum_{i=1}^{m} \operatorname{freq}\left(T_{i} \cup\left(x+T_{i}\right), \mathcal{T}\right) \cdot \operatorname{Vol}\left(A_{i}\right)>0,
\end{aligned}
$$

where $\left\{F_{n}\right\}_{n \geq 1}$ is a van Hove sequence, the $T_{i}$ 's are representatives of all tile types, and the $A_{i}$ 's are their supports. For this reason we may call the elements of $\Xi(\mathcal{T})$ the almost-periods of $\mathcal{T}$. Of course an almost-period only really "looks like" a period if the corresponding density dens $\left(D_{x}\right)$ is close to 1 .

Below we consider the cylinder set $X_{\{T\}, V}$ for a $\mathcal{T}$-tile $T$ and a Borel set $V$, which we denote $X_{T, V}$ to simplify the notation.

Lemma 4.4. Suppose $\Xi(\mathcal{T})$ is uniformly discrete. Then there is $r>0$ and $n_{0}>0$ such that for any Borel set $V$ with $\operatorname{diam}(V)<r$, all $x \in \Xi(\mathcal{T})$, and every $\mathcal{T}$-tile $T$,

$$
X_{T, V} \cap X_{Q^{n} x+T, V}=X_{T \cup\left(Q^{n} x+T\right), V} \quad \text { for all } n \geq n_{0} .
$$

Proof. Note that for any $x \in \Xi(\mathcal{T})$ there is $n_{0}>0$ such that $T \cup\left(Q^{n} x+T\right)$ is a $\mathcal{T}$-patch for all $n \geq n_{0}$, from the primitivity. We only need to check " $\subset$ " since the other inclusion is obvious in all cases. A tiling $\mathcal{S} \in X_{\mathcal{T}}$ is in the left-hand side of (4.3) if and only if there are two, possibly distinct, vectors $v_{1}, v_{2} \in V$ such that $-v_{1}+T \in \mathcal{S}$ and $-v_{2}+Q^{n} x+T \in \mathcal{S}$. Hence $v_{1}-v_{2}+Q^{n} x \in \Xi(\mathcal{S})=\Xi(\mathcal{T})$. However, $Q^{n} x \in \Xi(\mathcal{T})$ since $Q \Xi(\mathcal{T}) \subset \Xi(\mathcal{T})$. Since $\Xi(\mathcal{T})$ is uniformly discrete, $v_{1}-v_{2}=0$ if $\operatorname{diam}(V)$ is sufficiently small. Then $T \cup\left(Q^{n} x+T\right) \subset v_{1}+\mathcal{S}$, and hence $\mathcal{S}$ is in the right-hand side of (4.3). The lemma is proved. 
Proposition 4.5. Suppose that $\mathcal{T}$ is a repetitive fixed point of a primitive substitution with expansive map $Q$ such that $\Xi(\mathcal{T})$ is uniformly discrete and $\mathcal{T}$ has FLC. If $\left(X_{\mathcal{T}}, \mu, \mathbb{R}^{d}\right)$ has a pure discrete spectrum, then

$$
\lim _{n \rightarrow \infty} \operatorname{dens}\left(D_{Q^{n} x}\right)=1 \quad \text { for all } \quad x \in \Xi(\mathcal{T}) .
$$

Proof. Fix $x \in \Xi(\mathcal{T})$. By Theorem 4.3, for every eigenvalue $\alpha \in \mathbb{R}^{d}$ we have $e^{2 \pi i\left(Q^{n} x\right) \cdot \alpha} \rightarrow 1$. This implies

$$
\left(U_{Q^{n} x}-I\right) f_{\alpha} \rightarrow 0,
$$

in the norm of $L^{2}\left(X_{\mathcal{T}}, \mu\right)$, for the corresponding eigenfunction $f_{\alpha}$. Since $\left\|U_{Q^{n} x_{x}}-I\right\| \leq$ 2 , the sequence of operators $\left\{U_{Q^{n} x}-I\right\}_{n \geq 0}$ is uniformly bounded, so by (4.5) we have $U_{Q^{n} x} f \rightarrow f$ for any $f$ in the closed linear span of the eigenfunctions. Let $T$ be a $\mathcal{T}$-tile and let $V$ be a Borel set satisfying (4.3). Denote by $f$ the characteristic function of the cylinder set $X_{T, V}$, which is in the closed linear span of the eigenfunctions by assumption. We can write

$$
\begin{aligned}
\left\|U_{Q^{n} x} f-f\right\|_{2}^{2} & =\int_{X_{\mathcal{T}}}\left|f\left(-Q^{n} x+\mathcal{S}\right)-f(\mathcal{S})\right|^{2} d \mu(\mathcal{S}) \\
& =\mu\left(X_{T, V} \triangle X_{Q^{n} x+T, V}\right) \\
& =2\left[\mu\left(X_{T, V}\right)-\mu\left(X_{T, V} \cap X_{Q^{n} x+T, V}\right)\right] .
\end{aligned}
$$

The last equality uses the fact that $\mu$ is translation-invariant. It follows that

$$
\mu\left(X_{T, V} \cap X_{Q^{n} x+T, V}\right) \rightarrow \mu\left(X_{T, V}\right)
$$

for each $\mathcal{T}$-tile $T$.

Now, combining Lemma 4.4, (4.6), and Corollary 4.2, we obtain that

$$
\operatorname{freq}\left(T \cup\left(Q^{n} x+T\right), \mathcal{T}\right) \rightarrow \operatorname{freq}(T, \mathcal{T}), \quad \text { as } \quad n \rightarrow \infty,
$$

for any tile $T \in \mathcal{T}$. In view of (4.2) this implies that $\lim _{n \rightarrow \infty} \operatorname{dens}\left(D_{Q^{n} x}\right)=1$, and the proposition is proved.

Definition 4.6. A set $\Lambda \subset \mathbb{R}^{d}$ is a Meyer set if $\Lambda$ is Delone and there is a finite set $F$ so that $\Lambda-\Lambda \subset \Lambda+F$.

As the next theorem shows, under the additional assumption that $\Xi(\mathcal{T})$ is a Meyer set, the converse of Proposition 4.5 is also true. Notice that we do not add FLC to the assumption, since $\Xi(\mathcal{T})$ being a Meyer set implies it.

Theorem 4.7. Suppose that $\mathcal{T}$ is a repetitive fixed point of a primitive substitution with expansive map $Q$ such that $\Xi(\mathcal{T})$ is a Meyer set. Then $\left(X_{\mathcal{T}}, \mu, \mathbb{R}^{d}\right)$ has a pure discrete spectrum if and only if

$$
\lim _{n \rightarrow \infty} \operatorname{dens}\left(D_{Q^{n} x}\right)=1 \quad \text { for all } \quad x \in \Xi(\mathcal{T}) .
$$


This theorem extends Theorem 6.2 of [17] to the case of $d \geq 3$. Sufficiency was proved in Proposition 4.5. Necessity is not needed for our main results, so its proof (which is a modification of the proof in [17]) is given in the Appendix.

Remark 4.8. In this subsection we have been assuming that the fixed point $\mathcal{T}$ of our primitive substitution is repetitive. We note that this assumption is not restrictive if we are interested in the measure-theoretic dynamical system $\left(X_{\mathcal{T}}, \mu, \mathbb{R}^{d}\right)$. Indeed, if $\mathcal{T}$ is non-repetitive, then the inclusion mapping $X_{\mathcal{A}, \omega} \hookrightarrow X_{\mathcal{T}}$ induces an isomorphism of measure-preserving systems (this follows from the proofs in Section A.1). All the tilings in $X_{\mathcal{A}, \omega}$ are repetitive; further, we can find a periodic point for some power of $\omega$ and work with it.

\subsection{Pure-Pointedness on Delone Multisets}

Transferring the results of the previous subsection over to Delone multisets, we obtain three quick corollaries.

Corollary 4.9. If $\boldsymbol{\Lambda}$ is a primitive substitution Delone multiset with FLC such that every $\Lambda$-cluster is legal, then the dynamical system $\left(X_{\Lambda}, \mathbb{R}^{d}\right)$ is uniquely ergodic.

Proof. Apply Corollary 3.8 and Lemma 3.10 to Theorem 4.1.

Corollary 4.10. Suppose that $\Lambda=\left(\Lambda_{i}\right)_{i \leq m}$ is a primitive substitution Delone multiset with expansive map $Q$ such that $\Xi(\Lambda):=\bigcup\left(\Lambda_{i}-\Lambda_{i}\right)_{i \leq m}$ is a Meyer set and every $\Lambda$-cluster is legal. Then the dynamical system $\left(X_{\Lambda}, \mu, \mathbb{R}^{d}\right)$ has a pure discrete spectrum if and only if

$$
\lim _{n \rightarrow \infty} \operatorname{dens}\left(\boldsymbol{\Lambda} \cap\left(Q^{n} x+\boldsymbol{\Lambda}\right)\right)=\operatorname{dens}(\boldsymbol{\Lambda}) \quad \text { for all } \quad x \in \Xi(\boldsymbol{\Lambda}) .
$$

Proof. By Theorem $3.7 \Lambda$ is representable by a tiling $\mathcal{T}$. The legality of $\Lambda$ implies that of $\mathcal{T}$ and Lemma 3.3 shows that $\mathcal{T}$ is repetitive. The result is now a direct consequence of Theorem 4.7, in view of the fact that $\Xi(\mathcal{T})=\Xi(\boldsymbol{\Lambda})$.

Corollary 4.11. Suppose that $\boldsymbol{\Lambda}=\left(\Lambda_{i}\right)_{i \leq m}$ is a primitive substitution Delone multiset with expansive map $Q$ such that $\bigcup_{i=1}^{m} \Lambda_{i}$ lies in a lattice $L$ in $\mathbb{R}^{d}$ and every $\Lambda$-cluster is legal. Then the dynamical system $\left(X_{\Lambda}, \mu, \mathbb{R}^{d}\right)$ has pure discrete spectrum if and only if

$$
\lim _{n \rightarrow \infty} \operatorname{dens}\left(\Lambda \triangle\left(Q^{n} x+\boldsymbol{\Lambda}\right)\right)=0 \quad \text { for all } \quad x \in L^{\prime},
$$

where $L^{\prime}=L_{1}+\cdots+L_{m}$, and $L_{i}$ is the Abelian group generated by $\Lambda_{i}-\Lambda_{i}$ for $i \leq m$.

Proof. The Meyer set condition is obvious, since all the sets $\Lambda_{i}$ lie in the lattice $L$. We have $\boldsymbol{\Xi}(\boldsymbol{\Lambda})=\bigcup\left(\Lambda_{i}-\Lambda_{i}\right)_{i \leq m} \subset L^{\prime}$, so the necessity follows from Corollary 4.10. (This direction will not be needed for our main results in the next section though.) The condition (4.7) for $x \in \Lambda_{i}-\Lambda_{i}$ follows from Corollary 4.10. In order to prove it for all $x \in L^{\prime}$ we note that $\boldsymbol{\Lambda} \triangle(y+z+\boldsymbol{\Lambda}) \subset(\boldsymbol{\Lambda} \triangle(y+\boldsymbol{\Lambda})) \cup((y+\boldsymbol{\Lambda}) \triangle(y+z+\boldsymbol{\Lambda}))$ for 
any $y, z \in \mathbb{R}^{d}$, hence

$$
\operatorname{dens}(\boldsymbol{\Lambda} \Delta(y+z+\boldsymbol{\Lambda})) \leq \operatorname{dens}(\boldsymbol{\Lambda} \Delta(y+\boldsymbol{\Lambda}))+\operatorname{dens}(\boldsymbol{\Lambda} \Delta(z+\boldsymbol{\Lambda})),
$$

and the statement follows.

Remark 4.12. Note that the density in (4.7) does not depend on the choice of the van Hove sequence, since this is true for the density dens $\left(D_{Q^{n} x}\right)$ of the associated tiling by Corollary 4.2 and (4.2).

\section{Applications to Lattice Substitutions}

\subsection{Lattice Substitutions}

In what follows, $L$ will be a lattice in $\mathbb{R}^{d}$ and the mappings of $\Phi$ will always be affine linear mappings of the form $x \mapsto Q x+a$, where $Q \in \operatorname{End}_{\mathbb{Z}}(L)$ is the same for all the maps and $a \in L$. Such maps are restrictions of uniquely determined affine linear mappings on $\mathbb{R}^{d}$ and we will not distinguish them notationally. A mapping $Q \in \operatorname{End}_{\mathbb{Z}}(L)$ is called an inflation for $L$ if det $Q \neq 0$ and $\bigcap_{k=0}^{\infty} Q^{k} L=\{0\}$. So an expansive map $Q$ for $L$ is an inflation.

Definition 5.1. A substitution system on $L$ with inflation $Q$ is a pair $(\boldsymbol{\Lambda}, \Phi)$ consisting of

- a Delone multiset $\Lambda=\left(\Lambda_{i}\right)_{i \leq m}$, for which each $\Lambda_{i}$ is a subset of $L$ and all $\Lambda_{i}$ are mutually disjoint, and

- an $m \times m$ MFS $\Phi$ on $L$, for which

$$
\Lambda_{i}=\bigcup_{j \leq m} \bigcup_{f \in \Phi_{i j}} f\left(\Lambda_{j}\right), \quad i \leq m,
$$

where the maps of $\Phi$ are affine linear mappings of the form $x \mapsto Q x+a, a \in L$, and the unions in (5.1) are disjoint.

The substitution system $(\Lambda, \Phi)$ is primitive if $S(\Phi)$ is primitive. For any affine linear mapping $f: x \mapsto Q x+b$ on $L$ we denote the translational part, $b$, of $f$ by $t(f)$.

Let $(\Lambda, \Phi)$ be a primitive substitution system on $L$ with inflation $Q$. Then $\left(\Lambda, \Phi^{\ell}\right)$ is a primitive substitution system on $L$ with inflation $Q^{\ell}$, so without loss of generality we can assume that $S(\Phi)$ is a positive matrix. Let $L^{\prime}:=L_{1}+L_{2}+\cdots+L_{m}$, where $L_{i}:=\left\langle\Lambda_{i}-\Lambda_{i}\right\rangle$, i.e. the Abelian group generated by $\Lambda_{i}-\Lambda_{i}$.

Since $Q$ is an inflation for $L, Q$ is an inflation for $L^{\prime}$ also. In fact,

$$
\bigcup_{j \leq m} \bigcup_{f \in \Phi_{i j}}\left(Q\left(\Lambda_{j}\right)+t(f)\right)=\Lambda_{i}
$$

implies $\bigcup_{j \leq m} Q\left(\Lambda_{j}-\Lambda_{j}\right) \subset\left(\Lambda_{i}-\Lambda_{i}\right)$ for any $i \leq m$, and thus

$$
Q\left(L_{1}+\cdots+L_{m}\right) \subset L_{i} \quad \text { for any } \quad i \leq m .
$$

So $Q L^{\prime} \subset L^{\prime}$, hence $Q \in \operatorname{End}_{\mathbb{Z}}\left(L^{\prime}\right)$. Note also $\bigcap_{k=0}^{\infty} Q^{k} L^{\prime} \subset \bigcap_{k=0}^{\infty} Q^{k} L=\{0\}$. 
Note that $L / L^{\prime}$ is finite, since $\Lambda$ is a Delone multiset. Let $q:=|\operatorname{det} Q|=\left[L^{\prime}: Q L^{\prime}\right]$ $>1$. We define the $Q$-adic completion

$$
\bar{L}=(\bar{L})_{Q}=\lim _{\leftarrow k} L / Q^{k} L^{\prime}=\lim _{\leftarrow k}\left(\cdots \rightarrow L / Q^{k} L^{\prime} \rightarrow \cdots \rightarrow L / Q L^{\prime} \rightarrow L / L^{\prime}\right)
$$

of $L$ and

$$
\overline{L^{\prime}}=\left(\overline{L^{\prime}}\right)_{Q}=\lim _{\leftarrow k} L^{\prime} / Q^{k} L^{\prime}=\lim _{\leftarrow k}\left(\cdots \rightarrow L^{\prime} / Q^{k} L^{\prime} \rightarrow \cdots \rightarrow L^{\prime} / Q L^{\prime}\right)
$$

of $L^{\prime}$. Each of $\bar{L}$ and $\overline{L^{\prime}}$ will be supplied with the usual topology of a profinite group. We can identify $\bar{L}$ and $\left(L / L^{\prime}\right) \times \overline{L^{\prime}}$ as topological spaces. In particular, the cosets $a+Q^{k} \overline{L^{\prime}}, a \in L, k=1,2, \ldots$, form a basis of open sets of $\bar{L}$ and each of these cosets is open and closed. When we use the word coset in this paper, we mean either a coset of the form $a+Q^{k} \overline{L^{\prime}}$ in $\bar{L}$ or $a+Q^{k} L^{\prime}$ in $L$ according to the context. An important observation is that any two cosets in $\bar{L}$ are either disjoint or one is contained in the other. The same applies to cosets of $L$.

We let $\mu$ denote the Haar measure on $\bar{L}$, normalized so that $\mu(\bar{L})=1$. Thus for cosets,

$$
\mu\left(a+Q^{k} \overline{L^{\prime}}\right)=\frac{1}{|\operatorname{det} Q|^{k} \cdot\left|L / L^{\prime}\right|}=\frac{1}{q^{k} \cdot\left|L / L^{\prime}\right|}, \quad k=1,2, \ldots
$$

From $\bigcap_{k=0}^{\infty} Q^{k} L^{\prime}=\{0\}$, we conclude that the mapping $x \rightarrow\left\{x \bmod Q^{k} L^{\prime}\right\}_{k}$ embeds $L$ in $\bar{L}$. We identify $L$ with its image in $\bar{L}$ and note that $\bar{L}$ is then the closure of $L$. With this identification, $L$ is a dense subgroup of $\bar{L}$, so we have a unique extension of $\Phi$ to an MFS on $\bar{L}$. Thus if $f \in \Phi_{i j}$ and $f: x \mapsto Q x+a$, this formula defines a mapping on $\bar{L}$, to which we give the same name. Furthermore, defining the compact subsets in $\bar{L}$,

$$
W_{i}:=\overline{\Lambda_{i}}, \quad i \leq m,
$$

and using the relations (5.1) and the continuity of mappings, we have

$$
W_{i}=\bigcup_{j \leq m} \bigcup_{f \in \Phi_{i j}} f\left(W_{j}\right), \quad i \leq m .
$$

We call $(\mathbf{W}, \Phi)$ the associated $Q$-adic system.

Suppose $L=\bigcup_{i \leq m} \Lambda_{i}$. For any $i \leq m$, since $\left\langle\Lambda_{i}-\Lambda_{i}\right\rangle \subset L^{\prime}$, we have

$$
\Lambda_{i} \subset x+L^{\prime} \quad \text { for any } \quad x \in \Lambda_{i} \subset L .
$$

For $a \in L$, let

$$
\begin{aligned}
\Phi_{i j}[a]:= & \left\{f \in(\Phi)_{i j}: Q y+t(f) \equiv a \bmod Q L^{\prime},\right. \\
& \text { where } \left.f: x \mapsto Q x+t(f), \Lambda_{j} \subset y+L^{\prime}\right\} \\
= & \left\{f \in(\Phi)_{i j}: f\left(\Lambda_{j}\right) \subset a+Q L^{\prime}\right\} .
\end{aligned}
$$

Then

$$
\bigcup_{i, j \leq m} \bigcup_{f \in \Phi_{i j}[a]} f\left(\Lambda_{j}\right)=a+Q L^{\prime}
$$

Let $\Phi[a]:=\bigcup_{i, j \leq m} \Phi_{i j}[a]$. This partitions $\Phi$ into congruence classes induced by $L / Q L^{\prime}$. 
Definition 5.2. Let $(\boldsymbol{\Lambda}, \Phi)$ be a primitive substitution system on $L$ with inflation $Q$ and $L=\bigcup_{i \leq m} \Lambda_{i}$. We say that $(\Lambda, \Phi)$ admits a modular coincidence relative to $Q L^{\prime}$ if $\Phi[a]$ is contained entirely in one row of $\Phi$ for some $a \in L$.

It is easy to see that $(\Lambda, \Phi)$ admits a modular coincidence relative to $Q L^{\prime}$ if and only if $\left(a+Q L^{\prime}\right) \subset \Lambda_{i}$ for some $i \leq m$.

We define the notion of a model set (or cut and project set) [12].

Definition 5.3. A cut and project scheme (CPS) consists of a collection of spaces and mappings as follows:

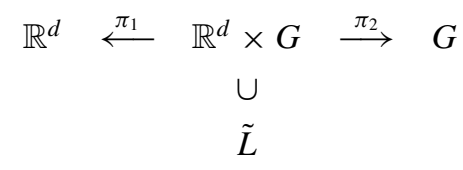

where $\mathbb{R}^{d}$ is a real Euclidean space, $G$ is some locally compact Abelian group, $\tilde{L} \subset$ $\mathbb{R}^{d} \times G$ is a lattice, i.e. a discrete subgroup for which the quotient group $\left(\mathbb{R}^{d} \times G\right) / \tilde{L}$ is compact, $\left.\pi_{1}\right|_{\tilde{L}}$ is injective, and $\pi_{2}(\tilde{L})$ is dense in $G$.

Definition 5.4. A model set in $\mathbb{R}^{d}$ is a subset of $\mathbb{R}^{d}$ which, up to translation, is of the form $\Gamma(V)=\left\{\pi_{1}(x): x \in \tilde{L}, \pi_{2}(x) \in V\right\}$ for some CPS as above, where $V \subset G$ has non-empty interior and compact closure (relatively compact). The model set $\Gamma(V)$ is regular if the boundary $\partial V=\bar{V} \backslash \stackrel{\circ}{V}$ of $V$ is of (Haar) measure 0 .

When we need to be more precise we explicitly mention the CPS from which a model set arises. This is quite important in some of the theorems below. Model sets are always Delone subsets of $\mathbb{R}^{d}$. We also find it convenient to consider certain degenerate types of model sets. A weak model set is a set in $\mathbb{R}^{d}$ of the form $\Gamma(V)$ where we assume only that $V$ is relatively compact, but not that it has a non-empty interior. When $V$ has no interior, $\Gamma(V)$ is not necessarily relatively dense in $\mathbb{R}^{d}$ but regularity still means that the boundary of $V$ is of measure 0 .

\subsection{Pure Pointedness, Modular Coincidence, and Model Sets}

This subsection contains our main new result, namely, that in the setting of lattice substitution systems, a pure point diffraction spectrum implies that there is a model set realization. Precise conditions are given in Theorem 5.12, which incorporates earlier results and completes the circle of equivalences started in [10]. The key new ingredient of the proof is Theorem 5.6. Some of the arguments in this subsection are similar to the corresponding parts of [10]. However, there is an important distinction: here we have to do everything modulo the sublattice $L^{\prime}$. For instance, the notion of modular coincidence is not the same as in [10], $\bar{L}$ is different, etc.

Consider $(\boldsymbol{\Lambda}, \Phi)$ a primitive substitution system on $L$ with an expansive map $Q$. Since $Q$ is expansive (see (3.1)), for any bounded subset $S$ of $\mathbb{R}^{d}$ containing 0 as an interior point and $\lambda>1$, there is large enough $k_{0}$ so that $Q^{k} S \supset \lambda S$ for $k>k_{0}$. 
Recall that for any set $F \subset \mathbb{R}^{d}$ we have a cluster $F \cap \boldsymbol{\Lambda}=\left(F \cap \Lambda_{i}\right)_{i \leq m} \subset \boldsymbol{\Lambda}$.

Suppose $0 \in \boldsymbol{\Lambda}$. Let $\mathbf{I}=\left(I_{i}\right)_{i \leq m}:=\Phi(0)=\left(\Phi_{i j}(0)\right)_{i \leq m}$ where $0 \in \Lambda_{j}$, and let $t(\Phi):=\left\{t(f): f \in \Phi_{i j}, f: x \mapsto Q x+t(f), i, j \leq m\right\}$. For a cluster $\mathbf{P}=\left(P_{i}\right)_{i \leq m}$ we write $\operatorname{supp}(\mathbf{P})=\bigcup_{i \leq m} P_{i}$. Let $\left\{\beta_{i}: i=1, \ldots, d\right\}$ be a basis of $L^{\prime}$. Let $D_{0}$ be the parallelepiped in $\mathbb{R}^{d}$ of the form

$$
D_{0}=\left\{x_{1} \beta_{1}+\cdots+x_{i} \beta_{i}+\cdots+x_{d} \beta_{d}:-1 \leq x_{i}<1,1 \leq i \leq d\right\} .
$$

We can always find $p \in \mathbb{Z}_{+}$so that $S\left(\Phi^{p}\right)$ is positive and $Q^{p} D_{0} \supset \lambda D_{0}$ for some $\lambda>1$, and then $a>0$ so that $D:=a D_{0} \supset\left(\operatorname{supp}\left(\Phi^{p}(0)\right) \cup t\left(\Phi^{p}\right)\right)$. Replacing $\Phi$ by $\Phi^{p}, Q D_{0}$ by $Q^{p} D_{0}$, etc., we may, for the purposes of Theorem 5.6, assume at the outset that $S(\Phi)$ is positive, $Q(D) \supset \lambda D$, and $D \supset(\operatorname{supp}(\mathbf{I}) \cup t(\Phi))$ for some $\lambda>1$ and convex $D$.

Lemma 5.5. Let $(\Lambda, \Phi)$ be a primitive substitution system on $L$ with expansive map $Q$ and $0 \in \Lambda$. Let $D$ be a convex set for which $Q(D) \supset \lambda D$ for some $\lambda>1$ and $D \supset(\operatorname{supp}(\mathbf{I}) \cup t(\Phi))$. Then there is $r>0$ such that $\operatorname{supp}\left(\Phi^{n}(\mathbf{I})\right) \subset Q^{n}(r D)$ for all $n \in \mathbb{Z}_{+}$.

Proof. Choose $p \in \mathbb{Z}_{+}$so that $\lambda^{p} \geq p+1$. Since $\lambda D \subset Q(D)$, we have $(p+1) D \subset$ $\lambda^{p} D \subset Q^{p}(D)$. So

$$
(p+1) D \subset Q^{p}(D) .
$$

Note that $Q(D) \supset D$ and $k D=\underbrace{D+\cdots+D}_{k}$ for any $k \in \mathbb{Z}_{+}$, since $D$ is convex.

Since we have $\operatorname{supp}(\mathbf{I}) \subset D$, which means $\mathbf{I} \subset D \cap \boldsymbol{\Lambda}$, for any $n \in \mathbb{Z}_{+}$,

$$
\begin{aligned}
\operatorname{supp} & \left(\Phi^{n}(\mathbf{I})\right) \\
& \subset \operatorname{supp}\left(\Phi^{n}(D \cap \boldsymbol{\Lambda})\right) \\
& \subset t(\Phi)+Q\left(\operatorname{supp}\left(\Phi^{n-1}(D \cap \boldsymbol{\Lambda})\right)\right) \\
& \subset t(\Phi)+\cdots+Q^{n-1}(t(\Phi))+Q^{n}(D) \\
& \subset D+\cdots+Q^{n-1}(D)+Q^{n}(D) .
\end{aligned}
$$

Since $Q(D) \supset \lambda D \supset D$, we have $Q^{i}(D) \supset Q^{j}(D)$ for $i>j$. Thus, writing $n=l p+s$, where $1 \leq s \leq p$ and $l \in \mathbb{Z}_{\geq 0}$, we obtain

$$
\begin{aligned}
\sum_{i=0}^{n} Q^{i}(D) & =\sum_{i=0}^{n-l p} Q^{i}(D)+\sum_{k=1}^{l} Q^{n-k p}\left(\sum_{i=1}^{p} Q^{i}(D)\right) \\
& \subset(p+1) Q^{n-l p}(D)+\sum_{k=1}^{l} Q^{n-k p}\left(p Q^{p}(D)\right) \\
& =Q^{n-l p}\left((p+1) D+p \sum_{k=1}^{l} Q^{(l-k+1) p}(D)\right) \\
& \subset Q^{n-l p}\left(Q^{p}(D)+p \sum_{j=1}^{l} Q^{j p}(D)\right) \quad \text { from (5.7). }
\end{aligned}
$$


So

$$
\begin{aligned}
\sum_{i=0}^{n} Q^{i}(D) & \subset Q^{n-l p} Q^{p}\left((p+1) D+p \sum_{j=1}^{l-1} Q^{j p}(D)\right) \\
& \subset Q^{n-l p} Q^{p}\left(Q^{p}(D)+p \sum_{j=1}^{l-1} Q^{j p}(D)\right) \\
& \vdots \\
& \subset Q^{n-l p} \overbrace{Q^{p} \cdots Q^{p}}^{l}((p+1) D)=Q^{n}((p+1) D) .
\end{aligned}
$$

In view of (5.8) this implies that $r:=p+1$ satisfies the assertion of the lemma.

Theorem 5.6. Let $(\Lambda, \Phi)$ be a repetitive primitive substitution system on $L$ with expansive map $Q$ and $L=\bigcup_{i \leq m} \Lambda_{i}$. If $\operatorname{dens}\left(\Lambda \triangle\left(Q^{n} \alpha+\Lambda\right)\right) \stackrel{n \rightarrow \infty}{\longrightarrow} 0$ for all $\alpha \in L^{\prime}$, then a modular coincidence relative to $Q^{M} L^{\prime}$ occurs in $\Phi^{M}$ for some $M \in \mathbb{Z}_{+}$.

Proof. Suppose that for all $n \in \mathbb{N}, \Phi^{n}$ does not admit any modular coincidence relative to $Q^{n} L^{\prime}$. We assume that $S(\Phi)$ is a positive matrix without loss of the generality. Then the cluster $\mathbf{I}=\left(I_{i}\right)_{i \leq m}=\left(\Phi_{i j}(0)\right)_{i \leq m}$, with $0 \in \Lambda_{j}$, has at least one element from each point set in $\boldsymbol{\Lambda}=\left(\Lambda_{i}\right)_{i \leq m}$. We claim that for all $n \in \mathbb{Z}_{+}$, $\operatorname{supp}\left(\Phi^{n}(\mathbf{I})\right)$ intersects every coset $x+Q^{n} L^{\prime}$ of $Q^{n} L^{\prime}$ in $L$ non-trivially. Indeed, $L=\bigcup_{i \leq m} \Lambda_{i}$ and each $\Lambda_{i} \subset I_{i}+L^{\prime}$ for $i \leq m$. So

$$
\begin{aligned}
L & =\bigcup_{j \leq m} \bigcup_{i \leq m}\left(\Phi^{n}\right)_{j i}\left(I_{i}+L^{\prime}\right) \\
& =\bigcup_{j \leq m} \bigcup_{i \leq m}\left(\left(\Phi^{n}\right)_{j i}\left(I_{i}\right)+Q^{n} L^{\prime}\right) \\
& =\operatorname{supp}\left(\Phi^{n}(\mathbf{I})\right)+Q^{n} L^{\prime} .
\end{aligned}
$$

So the claim follows.

To test modular coincidence we need only know about the translation parts of $\Phi^{n}$ and to which coset of $L^{\prime}$ each $\Lambda_{i}$ belongs (see Definition 5.2 and (5.4)). Since $\mathbf{I}$ has at least one element of each color type $i=1, \ldots, m$, modular coincidence can be tested on $\Phi^{n}(\mathbf{I})$ : if $\left(a+Q^{n} L^{\prime}\right) \cap \operatorname{supp}\left(\Phi^{n}(\mathbf{I})\right) \subset \Lambda_{i}$ for some $i$, then it means all the mappings in $\Phi^{n}$ which contribute to produce points in $a+Q^{n} L^{\prime}$ lie on the $i$-row of $\Phi^{n}$. So $\Phi^{n}$ has modular coincidence relative to $Q^{n} L^{\prime}$.

Since $Q$ is an expansive map, there is a parallelepiped $D=a D_{0}$ for which $Q(D) \supset$ $\lambda D$ and $D \supset(\operatorname{supp}(\mathbf{I}) \cup t(\Phi))$ for some $\lambda>1$. Then by Lemma 5.5 there is $r>0$ such that $\operatorname{supp}\left(\Phi^{n}(\mathbf{I})\right) \subset Q^{n}(r D)$ for all $n \in \mathbb{Z}_{+}$.

We have assumed that there is no modular coincidence. Thus, for any $a \in L$ and any $n \geq 1$, there exists $i \leq m$ and

$$
x, y \in \operatorname{supp}\left(\Phi^{n}(\mathbf{I})\right) \cap\left(a+Q^{n} L^{\prime}\right) \quad \text { such that } \quad x \in \Lambda_{i}, \quad y \notin \Lambda_{i} .
$$


Let $\beta_{-j}=-\beta_{j}$ for $j=1, \ldots, d$. We can write $y-x$ as a non-negative integer linear combination of the vectors $Q^{n} \beta_{j}, j \in\{ \pm 1, \ldots, \pm d\}$. Now $Q^{n}(r D)$ is a parallelepiped generated by $Q^{n} \beta_{j}, 1 \leq j \leq d$, which contains $\operatorname{supp}\left(\Phi^{n}(\mathbf{I})\right)$. Then there exists a path $x=x_{1}, x_{2}, \ldots, x_{s}=y$ entirely in $Q^{n}(r D)$ whose steps are each of the form $x_{l+1}-x_{l}=Q^{n} \beta_{j},|j| \leq d$, and we see that there is a $x^{\prime} \in Q^{n}(r D) \cap\left(a+Q^{n} L^{\prime}\right) \cap$ $\Lambda_{i}$ such that $x^{\prime}+Q^{n} \beta_{j} \in\left(Q^{n}(r D) \cap\left(a+Q^{n} L^{\prime}\right)\right) \backslash \Lambda_{i}$ for some $j,|j| \leq d$. It follows that

$$
\sharp\left[\bigcup_{|j| \leq d}\left(\boldsymbol{\Lambda} \triangle\left(Q^{n} \beta_{j}+\boldsymbol{\Lambda}\right)\right) \cap Q^{n}(r D)\right] \geq\left|\operatorname{det} Q^{n}\right| \cdot\left|L / L^{\prime}\right|,
$$

since there are $\left|\operatorname{det} Q^{n}\right| \cdot\left|L / L^{\prime}\right|$ cosets of $Q^{n} L^{\prime}$ in $L$ and each of the cosets contributes at least one point to our count.

Furthermore, for a parallelepiped $c+Q^{n}(r D)$ containing $\operatorname{supp}\left(c+\Phi^{n}(\mathbf{I})\right)$, for which $c+\Phi^{n}(\mathbf{I})$ is a translate of $\Phi^{n}(\mathbf{I})$, the argument goes in the same way. For $x, y$ in (5.9) we have

$(x+c) \in \operatorname{supp}\left(c+\Phi^{n}(\mathbf{I})\right) \cap \Lambda_{i}, \quad(y+c) \in \operatorname{supp}\left(c+\Phi^{n}(\mathbf{I})\right) \backslash \Lambda_{i}, \quad y-x \in Q^{n} L^{\prime}$.

As above, this implies that for some $x^{\prime \prime} \in\left(c+Q^{n}(r D)\right) \cap\left(c+a+Q^{n} L^{\prime}\right) \cap \Lambda_{i}$ we have $x^{\prime \prime}+Q^{n} \beta_{j} \in\left(\left(c+Q^{n}(r D)\right) \cap\left(c+a+Q^{n} L^{\prime}\right)\right) \backslash \Lambda_{i}$ for some $j,|j| \leq d$. Thus, similarly to $(5.10)$,

$$
\sharp\left[\bigcup_{|j| \leq d}\left(\boldsymbol{\Lambda} \triangle\left(Q^{n} \beta_{j}+\mathbf{\Lambda}\right)\right) \cap\left(c+Q^{n}(r D)\right)\right] \geq\left|\operatorname{det} Q^{n}\right| \cdot\left|L / L^{\prime}\right| .
$$

Let

$$
H_{k}=\left\{x \in L: x+(r D \cap \mathbf{\Lambda}) \subset F_{k} \cap \mathbf{\Lambda}\right\}
$$

and let $\tilde{H}_{k}$ be a maximal set of $x \in H_{k}$ such that $(x+r D)$ are mutually disjoint. Since $Q$ is invertible, $Q^{n}(x+r D)$ and $Q^{n}(y+r D)$ are disjoint if and only if $x+r D$ and $y+r D$ are disjoint. So $\left\{Q^{n}(x+r D) \cap \Lambda: x \in \tilde{H}_{k}\right\}$ is a set of disjoint $\Lambda$-clusters, which need not be translates of each other. We claim that each of the clusters $Q^{n}(x+r D) \cap \boldsymbol{\Lambda}$, for $x \in \tilde{H}_{k}$, contains a translate of $\Phi^{n}(\mathbf{I})$. Indeed, if $x \in \tilde{H}_{k}$, then $x+\mathbf{I} \subset F_{k} \cap \mathbf{\Lambda}$ since $\mathbf{I} \subset r D \cap \boldsymbol{\Lambda}$. It follows that $\Phi^{n}(x+\mathbf{I}) \subset \Phi^{n}(\boldsymbol{\Lambda})=\boldsymbol{\Lambda}, \Phi^{n}(x+\mathbf{I})=Q^{n} x+\Phi^{n}(\mathbf{I})$ since $x+\mathbf{I}$ is a translate of the cluster $\mathbf{I}$, and

$$
\begin{aligned}
\operatorname{supp}\left(\Phi^{n}(x+\mathbf{I})\right) & =Q^{n} x+\operatorname{supp}\left(\Phi^{n}(\mathbf{I})\right) \\
& \subset Q^{n} x+Q^{n}(r D) \\
& =Q^{n}(x+r D) .
\end{aligned}
$$

This claim, together with (5.11), yields

$$
\sharp\left[\bigcup_{|j| \leq d}\left(\boldsymbol{\Lambda} \triangle\left(Q^{n} \beta_{j}+\mathbf{\Lambda}\right)\right) \cap Q^{n}\left(F_{k}\right)\right] \geq \sharp \tilde{H}_{k} \cdot\left|\operatorname{det} Q^{n}\right| \cdot\left|L / L^{\prime}\right| .
$$

Recall that

$$
\operatorname{freq}(r D \cap \boldsymbol{\Lambda}, \boldsymbol{\Lambda})=\lim _{k \rightarrow \infty} \frac{\sharp H_{k}}{\operatorname{Vol}\left(F_{k}\right)},
$$


see Definition 2.3. Let $e:=\operatorname{diam}(r D)$. The set $x+r D$, for $x \in L$, can intersect at most $\sharp\left((r D)^{+e} \cap L\right)$ translates $y+r D, y \in L$. Thus,

$$
\sharp \tilde{H}_{k} \geq \frac{\sharp H_{k}}{\sharp\left((r D)^{+e} \cap L\right)} .
$$

Combining this with (5.12) we obtain

$$
\frac{\sharp\left[\bigcup_{|j| \leq d}\left(\Lambda \triangle\left(Q^{n} \beta_{j}+\Lambda\right)\right) \cap Q^{n}\left(F_{k}\right)\right]}{\operatorname{Vol}\left(Q^{n}\left(F_{k}\right)\right)} \geq \frac{\sharp H_{k} \cdot\left|L / L^{\prime}\right|}{\operatorname{Vol}\left(F_{k}\right) \cdot \sharp\left((r D)^{e} \cap L\right)} .
$$

Letting $k \rightarrow \infty$, we conclude that

$$
\sum_{|j| \leq d} \operatorname{dens}\left(\boldsymbol{\Lambda} \triangle\left(Q^{n} \beta_{j}+\boldsymbol{\Lambda}\right)\right) \geq \frac{\operatorname{freq}(r D \cap \boldsymbol{\Lambda}, \boldsymbol{\Lambda})}{\sharp\left((r D)^{e} \cap L\right)} \cdot\left|L / L^{\prime}\right| .
$$

Note that when passing to the limit in the left-hand side we used the van Hove sequence $\left\{Q^{n} F_{k}\right\}_{k \geq 1}$. This is legitimate in view of Remark 4.12.

By assumption, dens $\left(\boldsymbol{\Lambda} \triangle\left(Q^{n} \alpha+\boldsymbol{\Lambda}\right)\right) \stackrel{n \rightarrow \infty}{\longrightarrow} 0$ for all $\alpha \in L^{\prime}$, and in particular

$$
\sum_{|j| \leq d} \operatorname{dens}\left(\boldsymbol{\Lambda} \triangle\left(Q^{n} \beta_{j}+\mathbf{\Lambda}\right)\right) \stackrel{n \rightarrow \infty}{\longrightarrow} 0 .
$$

However, freq $(r D \cap \boldsymbol{\Lambda}, \boldsymbol{\Lambda})>0$ by the repetitive property. This is a contradiction.

Theorem 5.7. Let $(\Lambda, \Phi)$ be a primitive substitution system on $L$ with inflation $Q$. Let $(\mathbf{W}, \Phi)$ be the corresponding associated $Q$-adic system. Suppose $\bar{L}=\bigcup_{i \leq m} W_{i}$. Then

(i) $S\left(\Phi^{r}\right)=(S(\Phi))^{r}$, for all $r \geq 1$;

(ii) $\mu\left(W_{i}\right)=\left(1 / q^{r}\right) \sum_{j \leq m}\left(S\left(\Phi^{r}\right)\right)_{i j} \mu\left(W_{j}\right)$, for all $i \leq m, r \geq 1$;

(iii) $\stackrel{\circ}{W}_{i} \neq \emptyset$ and $\mu\left(\partial W_{i}\right)=0$, for all $i \leq m$.

Proof. For every measurable set $E \subset \bar{L}$ and any $f \in \Phi_{i j}$ where $f: x \mapsto Q x+a$, $\mu(f(E))=\mu(a+Q(E))=(1 /|\operatorname{det} Q|) \mu(E)$. In particular, $\mu\left(f\left(W_{j}\right)\right)=(1 / q) w_{j}$, where $w_{j}:=\mu\left(W_{j}\right)$ and $q=|\operatorname{det} Q|$. We obtain

$$
w_{i} \leq \sum_{j=1}^{m} \frac{1}{q^{r}}\left(S\left(\Phi^{r}\right)_{i j}\right) w_{j} \quad \text { for any } \quad r \geq 1
$$

from (5.3).

Let $w=\left(w_{i}\right)_{i \leq m}$. Since $\bar{L}=\bigcup_{i \leq m} W_{i}$, the Baire category theorem assures that for at least one $i$,

$$
\stackrel{\circ}{W_{i}} \neq \emptyset
$$

and then the primitivity gives this for all $i$. So $w>0$ and

$$
w \leq \frac{1}{q^{r}} S\left(\Phi^{r}\right) w \leq \frac{1}{q^{r}} S(\Phi)^{r} w \quad \text { for any } \quad r \geq 1 .
$$


Since $S(\Phi)^{r}$ is primitive and the PF eigenvalue of $S(\Phi)^{r}$ is $q^{r}=|\operatorname{det} Q|^{r}$ by [9], we have from Lemma 1 of [10] that

$$
w=\frac{1}{q^{r}} S\left(\Phi^{r}\right) w=\frac{1}{q^{r}} S(\Phi)^{r} w \quad \text { for any } \quad r \geq 1 .
$$

The positivity of $w$ together with $S\left(\Phi^{r}\right) \leq S(\Phi)^{r}$ shows that $S\left(\Phi^{r}\right)=S(\Phi)^{r}$. This proves (i) and (ii).

Fix any $i \leq m$, let $\stackrel{\circ}{W_{i}}$ contain a basis open set $a+Q^{r} \overline{L^{\prime}}$ with some $r \in \mathbb{Z}_{\geq 0}$ by (5.13). Since $\left(\Lambda, \Phi^{r}\right)$ is a substitution system, $a+Q^{r} \overline{L^{\prime}} \subset \stackrel{\circ}{W}_{i} \subset W_{i}=\bigcup_{j \leq m}\left(\Phi^{r}\right)_{i j} W_{j}$. In particular, $\left(a+Q^{r} \overline{L^{\prime}}\right) \cap g\left(W_{k}\right) \neq \emptyset$ for some $k \leq m$ and some $g \in\left(\Phi^{r}\right)_{i k}$. However, $g\left(z+\overline{L^{\prime}}\right)=Q^{r}\left(z+\overline{L^{\prime}}\right)+t(g)$, where $\Lambda_{k} \subset z+L^{\prime}$. So $\left(a+Q^{r} \overline{L^{\prime}}\right) \cap\left(t(g)+Q^{r} z+Q^{r} \overline{L^{\prime}}\right) \neq$ $\emptyset$. This means $a+Q^{r} \overline{L^{\prime}}=t(g)+Q^{r} z+Q^{r} \overline{L^{\prime}}$. Thus

$$
g\left(W_{k}\right) \subset g\left(z+\overline{L^{\prime}}\right)=a+Q^{r} \overline{L^{\prime}} \subset \stackrel{\circ}{W_{i}} .
$$

For all $f \in\left(\Phi^{r}\right)_{i j}, j \leq m, f$ is clearly an open map, so $\bigcup_{j \leq m}\left(\Phi^{r}\right)_{i j}\left(\stackrel{\circ}{W_{j}}\right) \subset \stackrel{\circ}{W_{i}}$. Thus

$$
\begin{aligned}
\partial W_{i}=W_{i} \backslash \stackrel{\circ}{W}_{i} & =\left(\bigcup_{j \leq m}\left(\Phi^{r}\right)_{i j}\left(W_{j}\right)\right) \backslash \stackrel{\circ}{W_{i}} \\
& \subset \bigcup_{j \leq m}\left(\left(\Phi^{r}\right)_{i j}\left(W_{j}\right) \backslash\left(\Phi^{r}\right)_{i j}\left(\stackrel{W}{j}_{j}\right)\right) \\
& \subset \bigcup_{j \leq m}\left(\Phi^{r}\right)_{i j}\left(\partial W_{j}\right) .
\end{aligned}
$$

Note that due to (5.16) at least one $g$ in $\left(\Phi^{r}\right)_{i j}$ does not contribute to the relation (5.17).

Let $v_{i}:=\mu\left(\partial W_{i}\right), i \leq m$, and $v:=\left(v_{i}\right)_{i \leq m}$. So $v \leq\left(1 / q^{r}\right) S\left(\Phi^{r}\right) v$. Actually, by what we just said,

$$
0 \leq v \leq \frac{1}{q^{r}} S^{\prime} v \leq \frac{1}{q^{r}} S\left(\Phi^{r}\right) v=\frac{1}{q^{r}} S(\Phi)^{r} v,
$$

where $S^{\prime} \leq S(\Phi)^{r}, S^{\prime} \neq S(\Phi)^{r}$. Now applying Lemma 1 of [10] again we obtain equality throughout (5.18). However, by Lemma 2 of [10] the eigenvalues of $\left(1 / q^{r}\right) S^{\prime}$ are strictly less in absolute value than the PF eigenvalue of $\left(1 / q^{r}\right) S(\Phi)^{r}$, which is 1 . This forces $v=0$, and hence $\mu\left(\partial W_{i}\right)=0, i \leq m$.

Theorem 5.8. Let $(\boldsymbol{\Lambda}, \Phi)$ be a primitive substitution system on $L$ with inflation $Q$ and $L=\bigcup_{i<m} \Lambda_{i}$. Let $W_{i}=\overline{\Lambda_{i}}$ in $\bar{L}$ for any $i \leq m$. If there is a modular coincidence relative to $Q^{M} L^{\prime}$ in $\Phi^{M}$, then $\stackrel{\circ}{W}_{s} \cap \stackrel{\circ}{W}_{t}=\emptyset$, where $s, t \leq m, s \neq t$.

Proof. By assumption, there is $\Lambda_{i}$ such that

$$
a+Q^{M} L^{\prime}=\bigcup_{j \leq m} \bigcup_{f \in\left(\Phi^{M}\right) i j[a]} f\left(\Lambda_{j}\right) \subset \Lambda_{i} \quad \text { for some } a \in L .
$$


Assume $\left(\Phi^{M}\right)_{i k}[a] \neq \emptyset$ for some $k$. Pick $f \in\left(\Phi^{M}\right)_{i k}[a]$, where $f: x \mapsto Q^{M} x+t(f)$. Then $Q^{M} y+t(f) \equiv a \bmod Q^{M} L^{\prime}$, where $\Lambda_{k} \subset y+L^{\prime}$, and $f\left(\Lambda_{k}\right) \subset a+Q^{M} L^{\prime}$.

Take any $s \leq m$ and suppose $\Lambda_{s} \subset z+L^{\prime}$. Pick $g \in \Phi_{k s} \neq \emptyset$, where $g: x \mapsto$ $Q x+t(g)$. Then

$$
\begin{aligned}
f \circ g\left(\Lambda_{s}\right) & =Q^{M}\left(Q\left(\Lambda_{s}\right)+t(g)\right)+t(f) \\
& =Q^{M+1}\left(\Lambda_{s}\right)+Q^{M}(t(g))+t(f) \\
& \subset Q^{M+1}(z)+Q^{M}(t(g))+t(f)+Q^{M+1} L^{\prime} .
\end{aligned}
$$

Let $c:=Q^{M+1}(z)+Q^{M}(t(g))+t(f)$. So

$$
f \circ g\left(\Lambda_{s}\right) \subset c+Q^{M+1} L^{\prime}
$$

Let $p:=f \circ g \in\left(\Phi^{M+1}\right)_{i s}$. Since $f \circ g\left(\Lambda_{s}\right) \subset a+Q^{M} L^{\prime} \subset \Lambda_{i}$,

$$
\left(a+Q^{M} L^{\prime}\right) \cap\left(c+Q^{M+1} L^{\prime}\right) \neq \emptyset .
$$

Thus

$$
c+Q^{M+1} L^{\prime} \subset a+Q^{M} L^{\prime} \subset \Lambda_{i}
$$

Let

$$
H_{j}:=\left\{h \in\left(\Phi^{M+1}\right)_{i j}: h\left(\Lambda_{j}\right) \subset c+Q^{M+1} L^{\prime}\right\}, \quad \text { where } \quad j \leq m .
$$

So

$$
c+Q^{M+1} L^{\prime}=\bigcup_{j \leq m} \bigcup_{h \in H_{j}} h\left(\Lambda_{j}\right) .
$$

Note that for any $j \leq m$ and $h \in H_{j}, Q^{M+1} x+t(h) \equiv c \bmod Q^{M+1} L^{\prime}$, where $\Lambda_{j} \subset$ $x+L^{\prime}$. So we can write (5.20) more explicitly as follows:

$$
\begin{aligned}
c+Q^{M+1} L^{\prime}=\bigcup_{\substack{j \leq m \\
h \in H_{j}}}\left\{c+Q^{M+1} \alpha_{h}+Q^{M+1} \Lambda_{j}:\right. \\
\left.c+Q^{M+1} \alpha_{h}=t(h), \text { where } \alpha_{h} \in L\right\} .
\end{aligned}
$$

So

$$
L^{\prime}=\bigcup_{j \leq m} \bigcup_{h \in H_{j}}\left(\alpha_{h}+\Lambda_{j}\right)
$$

Note $\alpha_{p}+\Lambda_{s} \subset L^{\prime}$. Separating off $\Lambda_{s}$, we get

$$
-\alpha_{p}+L^{\prime}=\Lambda_{s} \cup\left(\bigcup_{j \leq m} \bigcup_{h \in H_{j}^{\prime}}\left(-\alpha_{p}+\alpha_{h}+\Lambda_{j}\right)\right),
$$

where $H_{j}^{\prime}:=H_{j}$ if $j \neq s$, and $H_{s}^{\prime}:=H_{s} \backslash\{p\}$. Note that the decompositions of (5.22) are disjoint. However, we also know that $\Lambda_{s}$ and $\bigcup\left\{\Lambda_{j}: \Lambda_{j} \subset-\alpha_{p}+L^{\prime}, j \neq s\right\}$ are disjoint. So it follows that

$$
\bigcup\left\{\Lambda_{j}: \Lambda_{j} \subset-\alpha_{p}+L^{\prime}, j \neq s\right\} \subset \bigcup_{j \leq m} \bigcup_{h \in H_{j}^{\prime}}\left(-\alpha_{p}+\alpha_{h}+\Lambda_{j}\right) .
$$


Taking closures to both sides of (5.23),

$$
\bigcup\left\{W_{j}: \Lambda_{j} \subset-\alpha_{p}+L^{\prime}, j \neq s\right\} \subset \bigcup_{j \leq m} \bigcup_{h \in H_{j}^{\prime}}\left(-\alpha_{p}+\alpha_{h}+W_{j}\right) .
$$

On the other hand, if we apply Theorem 5.7 to $\Phi^{M+1}$ and look at (5.21), we get

$$
\mu\left(c+Q^{M+1} \overline{L^{\prime}}\right)=\sum_{j \leq m} \sum_{h \in H_{j}} \mu\left(Q^{M+1}\left(\alpha_{h}+W_{j}\right)+c\right) .
$$

Hence

$$
\mu\left(\overline{L^{\prime}}\right)=\sum_{j \leq m} \sum_{h \in H_{j}} \mu\left(\alpha_{h}+W_{j}\right)
$$

So

$$
\mu\left(-\alpha_{p}+\overline{L^{\prime}}\right)=\sum_{j \leq m} \sum_{h \in H_{j}} \mu\left(-\alpha_{p}+\alpha_{h}+W_{j}\right)
$$

Thus

$$
\mu\left(-\alpha_{p}+\overline{L^{\prime}}\right)=\mu\left(W_{s}\right)+\left(\sum_{j \leq m} \sum_{h \in H_{j}^{\prime}}-\alpha_{p}+\alpha_{h}+\mu\left(W_{j}\right)\right)
$$

which, after taking closures in (5.22), gives us

$$
\mu\left(W_{s} \cap\left(\bigcup_{j \leq m} \bigcup_{h \in H_{j}^{\prime}}\left(-\alpha_{p}+\alpha_{h}+W_{j}\right)\right)\right)=0 .
$$

Finally from (5.24) and (5.25) we obtain

$$
\mu\left(W_{s} \cap\left(\bigcup\left\{W_{j}: \Lambda_{j} \subset-\alpha_{p}+L^{\prime}, j \neq s\right\}\right)\right)=0 .
$$

It shows that $\stackrel{\circ}{W}_{s} \cap \stackrel{\circ}{W}_{j}=\emptyset$ for any $j$ with $\Lambda_{j} \subset-\alpha_{p}+L^{\prime}, j \neq s$. It is easy to see that $\stackrel{\circ}{W}_{s} \cap \stackrel{\circ}{W}_{k}=\emptyset$, where $\Lambda_{k} \not \subset-\alpha_{p}+L^{\prime}$. Since $s$ is arbitrary in $\{1, \ldots, m\}, \stackrel{\circ}{W}_{s} \cap \stackrel{\circ}{W}_{t}=\emptyset$ for all $s, t \leq m, s \neq t$.

Lemma 5.9. Let $\Lambda_{i}, i \leq m$, be point sets of the lattice $L$ in $\mathbb{R}^{d}$. Let $Q$ be an inflation of $L$, and identify $L$ and its image in $\bar{L}$. Define $W_{i}:=\overline{\Lambda_{i}}$ in $\bar{L}$ and $\Gamma_{i}:=W_{i} \cap L$.

(i) If $\Lambda_{1}, \ldots, \Lambda_{m}$ are disjoint and $\mu\left(\overline{\Gamma_{i} \backslash \Lambda_{i}}\right)=0$ for all $i \leq m$, then $\stackrel{\circ}{W}_{i} \cap \stackrel{\circ}{W}_{j}=\emptyset$ for all $i \neq j$.

(ii) If $L=\bigcup_{i \leq m} \Lambda_{i}$ and $\stackrel{\circ}{W_{i}} \cap \stackrel{\circ}{\Gamma_{j}}=\emptyset$ for all $i \neq j$, where $i, j \leq m$, then $\Gamma_{i} \backslash \Lambda_{i} \subset \bigcup_{j \leq m}^{\leq} \partial W_{j}$ for all $i \leq m$.

(iii) If $\mu\left(\partial W_{i}\right)=0$ for all $i \leq m$ and $\Gamma_{i} \backslash \Lambda_{i} \subset \bigcup_{j \leq m} \partial W_{j}$, then $\mu\left(\overline{\Gamma_{i} \backslash \Lambda_{i}}\right)=0$. 
Proof. (i) Suppose there are $i, j \leq m$ with $\stackrel{\circ}{W}_{i} \cap \stackrel{\circ}{W}_{j} \neq \emptyset$. We can choose $a \in$ $\left(\stackrel{\circ}{W}_{i} \cap \stackrel{\circ}{W}_{j}\right) \cap L$, since $L$ is dense in $\bar{L}$ and $\stackrel{\circ}{W}_{i} \cap \stackrel{\circ}{W}_{j}$ is open. Choose $k \in \mathbb{Z}_{+}$so that $a+Q^{k} \overline{L^{\prime}} \subset \stackrel{\circ}{W_{i}} \cap \stackrel{\circ}{W}_{j}$. Note that $a+Q^{k} L^{\prime} \subset \Gamma_{i} \cap \Gamma_{j}$. Then

$$
\begin{aligned}
\bigcup_{i \leq m}\left(\Gamma_{i} \backslash \Lambda_{i}\right) & \supseteq\left(\left(a+Q^{k} L^{\prime}\right) \backslash \Lambda_{i}\right) \cup\left(\left(a+Q^{k} L^{\prime}\right) \backslash \Lambda_{j}\right) \\
& \supseteq\left(a+Q^{k} L^{\prime}\right) \backslash\left(\Lambda_{i} \cap \Lambda_{j}\right) \\
& =a+Q^{k} L^{\prime}, \quad \text { since the } \Lambda_{i}, i \leq m, \text { are disjoint. }
\end{aligned}
$$

So

$$
\begin{aligned}
\sum_{i \leq m} \mu\left(\overline{\Gamma_{i} \backslash \Lambda_{i}}\right) & \geq \mu\left(\bigcup_{i \leq m}\left(\overline{\Gamma_{i} \backslash \Lambda_{i}}\right)\right) \\
& \geq \mu\left(a+Q^{k} \overline{L^{\prime}}\right) \\
& >0,
\end{aligned}
$$

contrary to assumption.

(ii) Assume $\stackrel{\circ}{W}_{i} \cap \stackrel{\circ}{W}_{j}=\emptyset$ for all $i \neq j$. For any $i \leq m$,

$$
\begin{aligned}
\left(\Gamma_{i} \backslash \Lambda_{i}\right) & \subset\left(\bigcup_{j \neq i} \Lambda_{j}\right) \cap W_{i} \quad\left(\text { since } L=\bigcup_{i \leq m} \Lambda_{i}\right) \\
& \subset \bigcup_{j \neq i}\left(W_{j} \cap W_{i}\right) \\
& \left.\subset \bigcup_{j \leq m} \partial W_{j} \quad \text { (since } \stackrel{\circ}{W}_{i} \cap \stackrel{\circ}{W}_{j}=\emptyset \text { for all } i \neq j\right) .
\end{aligned}
$$

(iii) Obvious.

We consider the following CPS:

$$
\begin{array}{cccc}
\mathbb{R}^{d} & \stackrel{\pi_{1}}{\longleftarrow} \mathbb{R}^{d} \times \bar{L} \stackrel{\pi_{2}}{\longrightarrow} \bar{L} \\
\cup & & \\
L & \longleftarrow & \tilde{L} & \longrightarrow \\
t & \longleftarrow & (t, t) & \longrightarrow
\end{array}
$$

where $\tilde{L}:=\{(t, t): t \in L\} \subset \mathbb{R}^{d} \times \bar{L}$.

In fact, $\bar{L}$ is a compact abelian group and $\tilde{L} \subset \mathbb{R}^{d} \times \bar{L}$ is a lattice, i.e. a discrete subgroup for which the quotient group $\left(\mathbb{R}^{d} \times \bar{L}\right) / \tilde{L}$ is compact. Furthermore, $\left.\pi_{1}\right|_{\tilde{L}}$ is injective and $\pi_{2}(\tilde{L})$ is dense in $\bar{L}$.

Theorem 5.10. Let $\Lambda_{i}, i \leq m$, be disjoint point sets of the lattice $L$ in $\mathbb{R}^{d}$. Identify $L$ and its image in $\bar{L}$. Let $W_{i}:=\overline{\Lambda_{i}}$ in $\bar{L}$ and $\Gamma_{i}:=W_{i} \cap L$. Suppose that $\mu\left(\partial W_{i}\right)=0$ for all $i \leq m$. 
(i) If $\Gamma_{i} \backslash \Lambda_{i} \subset \bigcup_{j \leq m} \partial W_{j}$, then, relative to the CPS (5.26), $\Lambda_{i}$ is a regular weak model set when $\stackrel{\circ}{W}_{i}$ is empty, and $\Lambda_{i}$ is a regular model set when $\stackrel{\circ}{W}_{i}$ is non-empty.

(ii) If $L=\bigcup_{j \leq m} \Lambda_{j}$ and each $\Lambda_{i}$ is a regular model set, then $\Gamma_{i} \backslash \Lambda_{i} \subset \bigcup_{j \leq m} \partial W_{j}$ for all $i \leq m$.

Proof. (i) Assume that $\Gamma_{i} \backslash \Lambda_{i} \subset \bigcup_{j \leq m} \partial W_{j}$ for all $i \leq m$. Since $\mu\left(\partial W_{i}\right)=0$ for all $i \leq m$,

$$
\mu\left(W_{i}\right)=\mu\left(\stackrel{\circ}{W}_{i}\right)=\mu\left(\stackrel{\circ}{W_{i}} \backslash \bigcup_{j \leq m} \partial W_{j}\right)
$$

Since $\Gamma_{i}=W_{i} \cap L$, we have $\Lambda_{i}=V_{i} \cap L$ where $V_{i}:=W_{i} \backslash\left(\Gamma_{i} \backslash \Lambda_{i}\right)$. Now $V_{i} \supset$

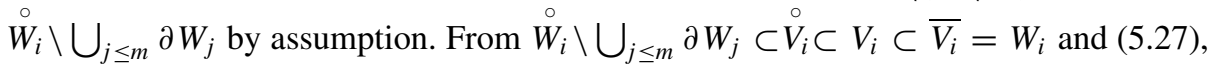
$\mu\left(\overline{V_{i}} \backslash \stackrel{\circ}{V}_{i}\right)=0$. So $\Lambda_{i}$ is regular. If $\stackrel{\circ}{W}_{i}=\emptyset$, then $\stackrel{\circ}{V}_{i}=\emptyset$ also. Thus $\Lambda_{i}$ is a regular weak model set. On the other hand, for any $i$ with $\stackrel{\circ}{W}_{i} \neq \emptyset$, we know that $\stackrel{\circ}{V}_{i} \neq \emptyset$ and $\overline{V_{i}}$ is compact. It follows that $\Lambda_{i}=V_{i} \cap L$ is a regular model set for the CPS (5.26).

(ii) Suppose that $\stackrel{\circ}{V}_{i} \neq \emptyset, \mu\left(\overline{V_{i}} \backslash \stackrel{\circ}{V}_{i}\right)=0$, where $\Lambda_{i}=V_{i} \cap L$, and $L=\bigcup_{j \leq m} \Lambda_{j}$. Then from $\overline{\Gamma_{i} \backslash \Lambda_{i}}=\overline{\left(W_{i} \cap L\right) \backslash\left(V_{i} \cap L\right)} \subset \overline{W_{i} \backslash V_{i}} \subset W_{i} \backslash \stackrel{\circ}{V}_{i}=\overline{V_{i}} \backslash \stackrel{\circ}{V}_{i}$, we have $\mu\left(\overline{\Gamma_{i} \backslash \Lambda_{i}}\right)=0$ for all $i \leq m$. By Lemma 5.9(i) and (ii), $\Gamma_{i} \backslash \Lambda_{i} \subset \bigcup_{j \leq m} \partial W_{j}$ for all $i \leq m$.

Theorem 5.11 [16]. If $\Gamma \subset \mathbb{R}^{d}$ is a regular model set, then $\Gamma$ has a pure point diffraction spectrum, i.e. the Fourier transform of its volume averaged autocorrelation measure is a pure point measure.

This theorem was established for real internal spaces in [8] and in full generality, as stated here, in [16]. For a new simpler proof of this result see [2].

Gathering all the results, we can state the following theorem, which says, in particular, that $\boldsymbol{\Lambda}$ has a pure point diffraction spectrum if and only if each $\Lambda_{i}$ is a regular model set with respect to a certain CPS, and that generalizes Dekking's well-known criterion for pure point diffractivity to lattice substitutions in $\mathbb{R}^{d}$.

Theorem 5.12. Let $\Lambda$ be a Delone multiset with expansive map $Q$ such that $(\Lambda, \Phi)$ is a primitive substitution system, $L=\bigcup_{i \leq m} \Lambda_{i}$ for some lattice $L$ in $\mathbb{R}^{d}$, and every $\Lambda$-cluster is legal. Let $L^{\prime}=L_{1}+\cdots+L_{m}$, where $L_{i}=\left\langle\Lambda_{i}-\Lambda_{i}\right\rangle$. The following are equivalent:

(i) $\Lambda$ has a pure point diffraction spectrum.

(ii) $\Lambda$ has a pure point dynamical spectrum.

(iii) $\operatorname{dens}\left(\boldsymbol{\Lambda} \triangle\left(Q^{n} \alpha+\boldsymbol{\Lambda}\right)\right) \stackrel{n \rightarrow \infty}{\longrightarrow} 0$ for all $\alpha \in L^{\prime}$.

(iv) A modular coincidence relative to $Q^{M} L^{\prime}$ occurs in $\Phi^{M}$ for some $M$.

(v) Each $\Lambda_{i}$ is a regular model set for $i \leq m$, relative to the CPS (5.26). 
Proof. It is easy to see that $\Lambda$ has FLC, since $\Lambda$ lies in a lattice $L$ in $\mathbb{R}^{d}$, and that $\Lambda$ is repetitive and has UCF, since every $\Lambda$-cluster is legal. So the proof goes as follows:

(i) $\Leftrightarrow$ (ii) [11, Theorem 3.2].

(ii) $\Rightarrow$ (iii) Corollary 4.11, using only the sufficiency, which follows from Proposition 4.5.

(iii) $\Rightarrow$ (iv) Theorem 5.6.

(iv) $\Rightarrow$ (v) Theorems 5.7 and 5.8, Lemma 5.9, and Theorem 5.10(i).

(v) $\Rightarrow$ (i) Theorem 5.11.

Remark 5.13. If $\Lambda$ is representable, but not repetitive, then it still may be possible to use the criteria of Theorem 5.12 to check for pure point diffractivity, using the argument in Remark 4.8. One only has to find another $\Gamma \in X_{\Lambda}$ which does satisfy the conditions in Theorem 5.12, since $\boldsymbol{\Lambda}$ is pure point diffractive if and only if $\boldsymbol{\Gamma}$ is pure point diffractive (see Theorem 3.2 of [11]).

Example 5.14 (Substitution Delone multiset with modular coincidence). Consider a substitution defined by $a \rightarrow a b c, b \rightarrow d c b, c \rightarrow c d a$, and $d \rightarrow d a b$. We can consider a corresponding MFS $\Phi$ as follows;

$$
\Phi=\left(\begin{array}{cccc}
\{3 x\} & \emptyset & \{3 x+2\} & \{3 x+1\} \\
\{3 x+1\} & \{3 x+2\} & \emptyset & \{3 x+2\} \\
\{3 x+2\} & \{3 x+1\} & \{3 x\} & \emptyset \\
\varnothing & \{3 x\} & \{3 x+1\} & \{3 x\}
\end{array}\right)
$$

A Delone multiset $\Lambda=\left(\Lambda_{a}, \Lambda_{b}, \Lambda_{c}, \Lambda_{d}\right)$ generated from $(\{0\},\{-1\}, \emptyset, \emptyset)$ is fixed under $\Phi$. On the real line, $\Lambda$ looks like

\begin{tabular}{lllllllllllllllll}
$\cdots$ & -6 & -5 & -4 & -3 & -2 & -1 & 0 & 1 & 2 & 3 & 4 & 5 & 6 & 7 & 8 & $\cdots$ \\
\hline$\cdots$ & $c$ & $d$ & $a$ & $d$ & $c$ & $b$ & $a$ & $b$ & $c$ & $d$ & $c$ & $b$ & $c$ & $d$ & $a$ & $\cdots$
\end{tabular}

Note that $\left\langle\Lambda_{\alpha}-\Lambda_{\alpha}: \alpha \in\{a, b, c, d\}\right\rangle=2 \mathbb{Z}$ and that $\Lambda_{a} \subset 2 \mathbb{Z}, \Lambda_{b} \subset 1+2 \mathbb{Z}$, $\Lambda_{c} \subset 2 \mathbb{Z}$, and $\Lambda_{d} \subset 1+2 \mathbb{Z}$.

$$
\Phi\left(\begin{array}{c}
\Lambda_{a} \\
\Lambda_{b} \\
\Lambda_{c} \\
\Lambda_{d}
\end{array}\right) \subset\left(\begin{array}{ccccccc}
6 \mathbb{Z} & \cup & \emptyset & \cup & (6 \mathbb{Z}+2) & \cup & (6 \mathbb{Z}+4) \\
(6 \mathbb{Z}+1) & \cup & (6 \mathbb{Z}+5) & \cup & \emptyset & \cup & (6 \mathbb{Z}+5) \\
(6 \mathbb{Z}+2) & \cup & (6 \mathbb{Z}+4) & \cup & 6 \mathbb{Z} & \cup & \emptyset \\
\emptyset & \cup & (6 \mathbb{Z}+3) & \cup & (6 \mathbb{Z}+1) & \cup & (6 \mathbb{Z}+3)
\end{array}\right) .
$$

Since each of $\Phi[3]$ and $\Phi[5]$ lies in one row of $\Phi$, the modular coincidence is confirmed. By Theorem 5.12 the sets $\Lambda_{1}, \Lambda_{2}, \Lambda_{3}, \Lambda_{4}$ are pure point diffractive and regular model sets. 


\subsection{Concluding Remarks}

We are still left with some open questions around Theorem 5.12. The most important is the requirement of legality, which we use as a connecting link to employ the results for tilings. Is it sufficient that $\boldsymbol{\Lambda}$ is repetitive? Example 3.12 illustrates a case in which legality fails, but repetitivity holds. One can observe that each of the two point sets of which it is comprised is pure point diffractive, even though the asymptotic condition of (iii) in Theorem 5.12 is not satisfied for some elements of $L^{\prime}$.

The entire context of Theorem 5.12 is that of an underlying lattice. Are there computable conditions (like modular coincidence) for substitution Delone sets which link to pure point diffractivity when we move away from the lattice environment?

What is the true relationship of the two concepts of pure point diffractivity and regular model sets? Assuming, say, a strictly ergodic substitution system satisfying the Meyer set condition (which is automatic for lattices), are these two concepts equivalent?

\section{Acknowledgment}

The authors are grateful to the referees whose comments helped to clarify the structure of this paper.

\section{Appendix}

\section{A.1. Unique Ergodicity}

In this section we show that if $\mathcal{T}$ is a fixed point of a primitive substitution, then the tiling dynamical system $\left(X_{\mathcal{T}}, \mathbb{R}^{d}\right)$ is uniquely ergodic. This is a bit more general than Theorem 3.1 of [17] where it was also assumed that $\mathcal{T}$ is repetitive. The proof goes through establishing the existence of uniform patch frequencies (UPF), the analog of UCF, see Definition 2.3. We present complete details here, in part because the proof of UPF was omitted in [17], but we should note that in a similar but slightly different setting the existence of UPF was established in Proposition 1 of [7] (see also p. 182 of [7] for references to earlier results of this kind).

Throughout this section we fix $\mathcal{T}$-a tiling satisfying $\omega(\mathcal{T})=\mathcal{T}$ for a primitive tile-substitution $\omega$.

Lemma A.1 (see, e.g. Proposition 1.1 of [13]). Every prototile in a primitive tilesubstitution has the boundary of zero Lebesgue measure.

Lemma A.2. Let $\mathcal{T}$ be a fixed point of a substitution with expansive map $Q$. Then for any tile $T \in \mathcal{T}$ with $T=(A, i)$ for some $i \leq m,\left\{Q^{n} A\right\}_{n \geq 1}$ is a van Hove sequence.

Proof. This is pretty straightforward from Lemma A.1. 
The following is proved in [17]. Note that it does not require repetitivity. Below PF is an abbreviation for "Perron-Frobenius."

Corollary A.3. Let $\omega$ be a primitive tile-substitution with prototiles $T_{i}=\left(A_{i}, i\right)$, for $i \leq m$, and expansive map $Q$. Then the PF eigenvalue of the substitution matrix $S$ is $|\operatorname{det}(Q)|$ and the vector $\left(\operatorname{Vol}\left(A_{i}\right)\right)_{i \leq m}$ is a left PF eigenvector. Thus,

$$
\lim _{n \rightarrow \infty}|\operatorname{det}(Q)|^{-n}\left(S^{n}\right)_{i j}=r_{i} \operatorname{Vol}\left(A_{j}\right),
$$

where $\left(r_{i}\right)_{i \leq m}$ is the right $P F$ eigenvector of $S$ such that $\sum_{i=1}^{m} r_{i} \operatorname{Vol}\left(A_{i}\right)=1$.

Notation. For a patch $P$ and a bounded set $F \subset \mathbb{R}^{d}$ denote

$$
L_{P}(F)=\sharp\left\{g \in \mathbb{R}^{d}: g+P \subset \mathcal{T},(g+\operatorname{supp}(P)) \subset F\right\}
$$

and

$$
N_{P}(F)=\sharp\left\{g \in \mathbb{R}^{d}: g+P \subset \mathcal{T},(g+\operatorname{supp}(P)) \cap F \neq \emptyset\right\} .
$$

Let $V_{\min }$ and $V_{\max }$ be the minimal and maximal volumes of $\mathcal{T}$-tiles respectively, let $\|Q\|$ be the operator norm, and let $t_{\max }$ be the maximal diameter of $\mathcal{T}$-tiles.

Inflated Tilings. Given a tiling $\mathcal{T}$ and an expansive map $Q^{\prime}$ on $\mathbb{R}^{d}$ we let

$$
Q^{\prime} \mathcal{T}=\left\{\left(Q^{\prime}(\operatorname{supp}(T)), l(T)\right): T \in \mathcal{T}\right\} .
$$

In other words, we blow up the tiles and retain their labels. Usually we will take $Q^{\prime}=Q^{k}$. If $\mathcal{T}$ is a fixed point of $\omega$, then the tilings $Q^{k} \mathcal{T}, k=1,2, \ldots$, form an hierarchical family of order $k$ supertilings in the sense that every tile of a higher-order tiling can be decomposed into tiles of a lower-order tiling.

Lemma A.4. Let $\mathcal{T}$ be a fixed point of a primitive substitution. Let $F \subset \mathbb{R}^{d}$ be an arbitrary bounded set, and let $\left\{F_{n}\right\}_{n \geq 1}$ be a van Hove sequence in $\mathbb{R}^{d}$. Then for any $\mathcal{T}$-patch $P$ and any $h \in \mathbb{R}^{d}$ we have:

(i) $L_{P}(F) \leq c_{1} \operatorname{Vol}(F)$, where $c_{1}$ depends only on $\mathcal{T}$.

(ii) If $P$ is a legal patch, then there are $c_{2}, n_{0}>0$ (depending on $P$ and $\left\{F_{n}\right\}_{n \geq 1}$, but not on $h)$ so that $L_{P}\left(h+F_{n}\right) \geq c_{2} \operatorname{Vol}\left(F_{n}\right)$, for all $n \geq n_{0}$.

(iii) If $P$ is a legal patch, then $\lim _{n \rightarrow \infty} N_{P}\left(\partial\left(h+F_{n}\right)\right) / L_{P}\left(h+F_{n}\right)=0$ uniformly in $h$.

Proof. (i) Select any tile from the patch $P$. Then distinct $\mathcal{T}$-patches equivalent to $P$ will have distinct selected tiles. Therefore,

$$
\operatorname{Vol}(F) \geq L_{P}(F) V_{\min },
$$

so we can take $c_{1}=V_{\min }^{-1}$.

(ii) If $P$ is a legal patch, then its translate occurs in some patch $\omega^{k}\left(T_{i}\right)$. Let $\ell \in \mathbb{N}$ be such that $\omega^{\ell}\left(T_{j}\right)$ contains tiles of all types, for all $j \leq m$. (This exists by the primitivity 
of the substitution.) Then every patch $\omega^{k+\ell}(T), T \in \mathcal{T}$, contains a translate of $P$. We consider the supertiling $Q^{k+\ell} \mathcal{T}$. It follows that for any set $F, L_{P}(F)$ is at least the number of $Q^{k+\ell} \mathcal{T}$-tiles whose supports are contained in $F$. Therefore, for $r=\|Q\|^{k+\ell} \cdot t_{\max }$,

$$
\begin{aligned}
& L_{P}\left(h+F_{n}\right) \cdot|\operatorname{det}(Q)|^{k+\ell} V_{\max } \\
& \geq \operatorname{Vol}\left(h+F_{n}^{-r}\right)=\operatorname{Vol}\left(F_{n}^{-r}\right) \geq \operatorname{Vol}\left(F_{n}\right)-\operatorname{Vol}\left(\left(\partial F_{n}\right)^{+r}\right) .
\end{aligned}
$$

This implies the desired statement in view of (2.1).

(iii) Let $t=\operatorname{diam}(P)$. Then

$$
\frac{N_{P}\left(h+\partial F_{n}\right)}{L_{P}\left(h+F_{n}\right)} \leq \frac{L_{P}\left(h+\partial F_{n}{ }^{+t}\right)}{L_{P}\left(h+F_{n}\right)} \leq \frac{c_{1} \operatorname{Vol}\left(h+\partial F_{n}{ }^{+t}\right)}{c_{2} \operatorname{Vol}\left(h+F_{n}\right)} \rightarrow 0,
$$

from (i) and (ii).

Lemma A.5. Let $\mathcal{T}$ be a fixed point of a primitive substitution with expansive map $Q$. Let $P$ be a $\mathcal{T}$-patch. Then

$$
c_{P}:=\lim _{n \rightarrow \infty} \frac{L_{P}\left(Q^{n} A\right)}{\operatorname{Vol}\left(Q^{n} A\right)}
$$

exists uniformly in A, a support of a $\mathcal{T}$-tile.

Proof. If $P$ is non-legal, then $L_{P}\left(Q^{n} A\right)=0$ for every $n$ and every tile support $A$, so $c_{P}=0$.

Assume now that $P$ is a legal patch. Let $\left\{T_{1}, \ldots, T_{m}\right\}$ be representatives of all $\mathcal{T}$-tile types, having supports $A_{i}, i \leq m$. Fix $\varepsilon>0$. By Lemmas A.4(iii) and A.2, we can find $k_{0} \in \mathbb{N}$ so that for any $k \geq k_{0}$ and any tile support $A$ on $\mathcal{T}$,

$$
N_{P}\left(\partial Q^{k} A\right) \leq \varepsilon L_{P}\left(Q^{k} A\right) .
$$

Choose a tile $T \in \mathcal{T}$. Then $T=(A, j)$ for some $j \leq m$, where $A=\operatorname{supp}(T)$. Consider the subdivision of $Q^{n} A=\operatorname{supp}\left(Q^{n} T\right), n>k>k_{0}$, into the tiles of $Q^{k} \mathcal{T}$. By the definition of the substitution matrix $S$, there are $\left(S^{n-k}\right)_{i j}$ tiles equivalent to $Q^{k} T_{i}$ in the subdivision of $Q^{n} T$. Therefore, in view of (A.1),

$$
\sum_{i=1}^{m} L_{P}\left(Q^{k} A_{i}\right)\left(S^{n-k}\right)_{i j} \leq L_{P}\left(Q^{n} A\right) \leq(1+\varepsilon) \sum_{i=1}^{m} L_{P}\left(Q^{k} A_{i}\right)\left(S^{n-k}\right)_{i j} .
$$

By Corollary A.3,

$$
\lim _{n \rightarrow \infty} \frac{\left(S^{n-k}\right)_{i l}}{\operatorname{Vol}\left(Q^{n} A_{l}\right)}=r_{i}|\operatorname{det}(Q)|^{-k} \quad \text { for any } \quad l \leq m .
$$

Thus, dividing (A.2) by $\operatorname{Vol}\left(Q^{n} A\right)$ and letting $n \rightarrow \infty$ we obtain

$$
\limsup _{n \rightarrow \infty} \frac{L_{P}\left(Q^{n} A\right)}{\operatorname{Vol}\left(Q^{n} A\right)}-\liminf _{n \rightarrow \infty} \frac{L_{P}\left(Q^{n} A\right)}{\operatorname{Vol}\left(Q^{n} A\right)} \leq \varepsilon|\operatorname{det}(Q)|^{-k} \sum_{i=1}^{m} r_{i} L_{P}\left(Q^{k} A_{i}\right) .
$$


By Lemma A.4(i), the right-hand side does not exceed $c_{1} \varepsilon \sum_{i=1}^{m} r_{i} \operatorname{Vol}\left(A_{i}\right)=c_{1} \varepsilon$. Since $\varepsilon>0$ is arbitrary, this proves the existence of the limit. Moreover,

$$
\sum_{i=1}^{m} L_{P}\left(Q^{k} A_{i}\right) r_{i}|\operatorname{det}(Q)|^{-k} \leq c_{P} \leq(1+\varepsilon) \sum_{i=1}^{m} L_{P}\left(Q^{k} A_{i}\right) r_{i}|\operatorname{det}(Q)|^{-k}
$$

and it does not depend on the choice of the tile $T$ on $\mathcal{T}$. Since $L_{P}\left(Q^{k} A_{i}\right)>0$ for $k$ sufficiently large, we have $c_{P}>0$.

Lemma A.6. Let $\mathcal{T}$ be a fixed point of a primitive substitution. For any $\mathcal{T}$-patch $P$ and for any van Hove sequence $\left\{F_{n}\right\}_{n \geq 1}$, there exists

$$
\operatorname{freq}(P, \mathcal{T}):=\lim _{n \rightarrow \infty} \frac{L_{P}\left(h+F_{n}\right)}{\operatorname{Vol}\left(F_{n}\right)}=c_{P},
$$

uniformly in $h \in \mathbb{R}^{d}$.

Proof. Consider the decomposition of the space $\mathbb{R}^{d}$ into the tiles of $Q^{k} \mathcal{T}$ for $k$ large. Then $L_{P}\left(h+F_{n}\right)$ is roughly the sum of $L_{P}\left(Q^{k} A\right)$ where $Q^{k} A$ ranges over the supports of those $Q^{k} \mathcal{T}$-tiles which intersect $h+F_{n}$. For large $n$ the "boundary effects" from $\partial F_{n}$ become small by the definition of van Hove sequence. Note also that the "boundary effects" from $\partial Q^{k} A$ become small. This is the idea; now we give the details.

Let $G_{k, n}=\left\{A: Q^{k} A \cap\left(h+F_{n}\right) \neq \emptyset, A=\operatorname{supp}(T)\right.$ for $\left.T \in \mathcal{T}\right\}$ and $H_{k, n}=$ $\left\{A: Q^{k} A \subset\left(h+F_{n}\right), A=\operatorname{supp}(T)\right.$ for $\left.T \in \mathcal{T}\right\}$ for $k, n \geq 1$. We have

$$
\sum_{A \in H_{k, n}} L_{P}\left(Q^{k} A\right) \leq L_{P}\left(h+F_{n}\right) \leq \sum_{A \in G_{k, n}}\left[L_{P}\left(Q^{k} A\right)+N_{P}\left(\partial\left(Q^{k} A\right)\right)\right] .
$$

Fix $\varepsilon>0$. Using Lemmas A.5 and A.4(iii) choose $k$ so that for any tile support $A$,

$$
\left|L_{P}\left(Q^{k} A\right) / \operatorname{Vol}\left(Q^{k} A\right)-c_{P}\right|<\varepsilon \quad \text { and } \quad N_{P}\left(\partial\left(Q^{k} A\right)\right)<\varepsilon L_{P}\left(Q^{k} A\right) .
$$

Combining (A.4) and (A.5) we obtain

$$
\left(c_{P}-\varepsilon\right) \sum_{A \in H_{k, n}} \operatorname{Vol}\left(Q^{k} A\right) \leq L_{P}\left(h+F_{n}\right) \leq(1+\varepsilon)\left(c_{P}+\varepsilon\right) \sum_{A \in G_{k, n}} \operatorname{Vol}\left(Q^{k} A\right) .
$$

Let $t_{k}:=\max \left\{\operatorname{diam}\left(Q^{k} A\right): A=\operatorname{supp}(T), T \in \mathcal{T}\right\}$. Observe that

$$
\sum_{A \in H_{k, n}} \operatorname{Vol}\left(Q^{k} A\right) \geq \operatorname{Vol}\left(F_{n}^{-t_{k}}\right) \quad \text { and } \quad \sum_{A \in G_{k, n}} \operatorname{Vol}\left(Q^{k} A\right) \leq \operatorname{Vol}\left(F_{n}^{+t_{k}}\right) .
$$

Since $k$ is fixed, by the van Hove property we have for $n$ sufficiently large,

$$
\operatorname{Vol}\left(F_{n}^{-t_{k}}\right) \geq(1-\varepsilon) \operatorname{Vol}\left(F_{n}\right) \quad \text { and } \quad \operatorname{Vol}\left(F_{n}^{+t_{k}}\right) \leq(1+\varepsilon) \operatorname{Vol}\left(F_{n}\right) .
$$

Combining everything, we obtain for $n$ sufficiently large,

$$
\left(c_{P}-\varepsilon\right)(1-\varepsilon) \leq \frac{L_{P}\left(h+F_{n}\right)}{\operatorname{Vol}\left(F_{n}\right)} \leq\left(c_{P}+\varepsilon\right)(1+\varepsilon)^{2} .
$$

Since $\varepsilon$ was arbitrary, this implies (A.3) as desired. 


\section{A.2. Overlap Coincidence}

Here we prove the necessity in Theorem 4.7. We use the notion of overlaps and the subdivision graph of overlaps from p. 721 of [17], with some modifications.

Definition A.7. Let $\mathcal{T}$ be a tiling. A triple $(T, y, S)$, with $T, S \in \mathcal{T}$ and $y \in \Xi(\mathcal{T})$, is called an overlap if the intersection $\operatorname{supp}(y+T) \cap \operatorname{supp}(S)$ has non-empty interior. We say that two overlaps $(T, y, S)$ and $\left(T^{\prime}, y^{\prime}, S^{\prime}\right)$ are equivalent if for some $g \in \mathbb{R}^{d}$ we have $y+T=g+y^{\prime}+T^{\prime}, S=g+S^{\prime}$. Denote by $[(T, y, S)]$ the equivalence class of an overlap. An overlap $(T, y, S)$ is a coincidence if $y+T=S$. The support of an overlap $(T, y, S)$ is $\operatorname{supp}(T, y, S)=\operatorname{supp}(y+T) \cap \operatorname{supp}(S)$.

Lemma A.8. Let $\mathcal{T}$ be a tiling such that $\Xi(\mathcal{T})$ is a Meyer set. Then the number of equivalence classes of overlaps for $\mathcal{T}$ is finite.

Proof. Let $T_{i}, i \leq m$, be the representatives of all tile types for $\mathcal{T}$. Let $\Lambda_{i}$ be the Delone set such that $\Lambda_{i}+T_{i}$ is the collection of all tiles of type $i$. Thus we have $\mathcal{T}=\bigcup_{i \leq m}\left(\Lambda_{i}+T_{i}\right)$. Let $(T, y, S)$ be an overlap. We have $T=u_{i}+T_{i}$ and $S=u_{j}+T_{j}$ for some $i, j \leq m$ (possibly equal) and some $u_{i} \in \Lambda_{i}, u_{j} \in \Lambda_{j}$. The equivalence class of the overlap is completely determined by $i, j$, and the vector $u_{i}+y-u_{j}$. Since the interiors of the supports of $y+T$ and $S$ must intersect, we have

$$
\left|u_{i}+y-u_{j}\right| \leq C,
$$

where $C=2 \max \{\operatorname{diam}(T): T \in \mathcal{T}\}$. Note that $u_{i}, y, u_{j} \in \Xi$. By the definition of $\Xi:=\Xi(\mathcal{T})$ we have $\Xi=-\Xi$. By the definition of Meyer set, $\Xi-\Xi \subset \Xi+F$ for some finite set $F$. This implies $\Xi+\Xi-\Xi \subset \Xi+(F+F)$, which is a discrete set, so there are finitely many possible vectors $u_{i}+y-u_{j}$ in (A.6). This proves the lemma.

Next we define the subdivision graph $\mathcal{G}_{\mathcal{O}}(\mathcal{T})$ for overlaps. Its vertices are equivalence classes of overlaps. Let $\mathcal{O}=(T, y, S)$ be an overlap. We will specify directed edges leading from the equivalence class $[\mathcal{O}]$. Recall that we have the tile-substitution $\omega$, see Definition 3.1. Then $\omega(y+T)=Q y+\omega(T)$ is a patch of $Q y+\mathcal{T}$, and $\omega(S)$ is a $\mathcal{T}$-patch, and, moreover,

$$
\operatorname{supp}(Q y+\omega(T)) \cap \operatorname{supp}(\omega(S))=Q(\operatorname{supp}(T, y, S)) .
$$

For each pair of tiles $T^{\prime} \in \omega(T)$ and $S^{\prime} \in \omega(S)$ such that $\mathcal{O}^{\prime}:=\left(T^{\prime}, Q y, S^{\prime}\right)$ is an overlap, we draw a directed edge from $\left[\mathcal{O}^{\prime}\right]$ to $\left[\mathcal{O}^{\prime}\right]$.

Lemma A.9. Let $\mathcal{T}$ be a repetitive fixed point of a primitive substitution with expansive map $Q$ such that $\Xi(\mathcal{T})$ is a Meyer set. Let $x \in \Xi(\mathcal{T})$. The following are equivalent:

(i) $\lim _{n \rightarrow \infty} \operatorname{dens}\left(D_{Q^{n} x}\right)=1$.

(ii) $1-\operatorname{dens}\left(D_{Q^{n} x}\right) \leq C r^{n}, n \geq 1$, for some $C>0$ and $r \in(0,1)$.

(iii) From each vertex of the graph $\mathcal{G}_{\mathcal{O}}(\mathcal{T})$ there is a path leading to a coincidence. 
Proof. We have for each $n \geq 0$,

$$
\mathbb{R}^{d}=\bigcup_{T \in \mathcal{T}} \bigcup_{S \in \mathcal{T}} \operatorname{supp}\left(T, Q^{n} x, S\right),
$$

where the support is considered empty if $\left(T, Q^{n} x, S\right)$ is not an overlap. Notice that $\operatorname{supp}\left(D_{Q^{n_{x}}}\right)$ is exactly the union of supports of coincidences in this formula. It is clear that all edges from the overlaps-coincidences lead to other coincidences. Thus,

$$
Q\left(\operatorname{supp}\left(D_{Q^{n} x}\right)\right) \subset \operatorname{supp}\left(D_{Q^{n+1} x}\right)
$$

and

$$
\operatorname{dens}\left(D_{Q^{n} x}\right) \leq \operatorname{dens}\left(D_{Q^{n+1} x}\right) .
$$

Now, if (iii) holds, then there exists $\ell \in \mathbb{N}$ such that for each overlap $\mathcal{O}$ the "inflation" $Q^{\ell}(\operatorname{supp}(\mathcal{O}))$ contains a coincidence. The volume of the support of a coincidence is at least $V_{\min }$. Thus

$$
1-\operatorname{dens}\left(D_{Q^{n+\ell} x}\right) \leq\left(1-\frac{V_{\min }}{V_{\max }|\operatorname{det}(Q)|^{\ell}}\right)\left(1-\operatorname{dens}\left(D_{Q^{n} x}\right)\right) .
$$

For any $n \geq 0, n=k \ell+s$ for some $k \in \mathbb{N}$ and $0 \leq s<\ell$. So

$$
\begin{aligned}
1-\operatorname{dens}\left(D_{Q^{n} x}\right) & =1-\operatorname{dens}\left(D_{Q^{k \ell+s} x}\right) \\
& \leq b^{k}\left(1-\operatorname{dens}\left(D_{Q^{s} x}\right)\right) \quad\left(\text { where } b=1-\frac{V_{\min }}{V_{\max }|\operatorname{det}(Q)|^{\ell}}\right) \\
& =\left(b^{1 / \ell}\right)^{k \ell+s} \frac{\left(1-\operatorname{dens}\left(D_{Q^{s} x}\right)\right)}{b^{s / \ell}} \\
& \leq r^{n} C \quad \text { for some } \quad r \in(0,1) \quad \text { and } \quad C>0 .
\end{aligned}
$$

Then (ii) follows.

It is straightforward that (ii) implies (i).

It remains to prove that (i) implies (iii). Suppose to the contrary, that there is an overlap $\mathcal{O}$ from which there is no path to a coincidence. Then $Q^{n}(\operatorname{supp}(\mathcal{O})) \subset \mathbb{R}^{d} \backslash \operatorname{supp}\left(D_{Q^{n} x}\right)$ for all $n$. By the repetitivity of $\mathcal{T}$, the overlaps equivalent to $\mathcal{O}$ occur relatively dense in $\mathbb{R}^{d}$. Therefore,

$$
1-\operatorname{dens}\left(D_{Q^{n} x}\right) \geq \operatorname{dens}\left(Q^{n}(\operatorname{supp}(\mathcal{O}))=\operatorname{dens}(\operatorname{supp}(\mathcal{O}))>0,\right.
$$

which contradicts (i). This completes the proof of the lemma.

Proof of Theorem 4.7. The assumption gives us through Lemma A.9 that there exists a basis $\mathcal{B}$ for $\mathbb{R}^{d}$ such that for all $x \in \mathcal{B}$,

$$
\sum_{n=0}^{\infty}\left(1-\operatorname{dens}\left(D_{Q^{n} x}\right)\right)<\infty .
$$

Then Theorem 6.1 of [17] implies that the dynamical system $\left(X_{\mathcal{T}}, \mu, \mathbb{R}^{d}\right)$ has a pure discrete spectrum. 


\section{References}

1. M. Baake and R. V. Moody, Self-similar measures for quasi-crystals, in Directions in Mathematical Quasicrystals, M. Baake and R. V. Moody, eds., CRM Monograph Series, Vol. 13, AMS, Providence, RI, 2000, pp. 1-42.

2. M. Baake and R. V. Moody, Weighted Dirac combs with pure point diffraction, Preprint, 2002.

3. L. Danzer, Inflation-species of planar tilings which are not of locally finite complexity, Proc. Steklov Inst. Math. 239 (2002), 1-9.

4. M. Dekking, The spectrum of dynamical systems arising from substitutions of constant length, Z. Wahrsch. Verw. Gebiete 41 (1978), 221-239.

5. S. Dworkin, Spectral theory and X-ray diffraction, J. Math. Phys. 34 (1993), 2965-2967.

6. H. Furstenberg, Recurrence in Ergodic Theory and Combinatorial Number Theory, Princeton University Press, Princeton, NJ, 1981.

7. C. P. M. Geerse and A. Hof, Lattice gas models on self-similar aperiodic tilings, Rev. Math. Phys. 3 (1991), $163-221$.

8. A. Hof, Diffraction by aperiodic structures, in The Mathematics of Long-Range Aperiodic Order, R. V. Moody, ed., Kluwer, Dordrecht, 1997, pp. 239-268,

9. J. C. Lagarias and Y. Wang, Substitution Delone sets, Discrete Comput. Geom. 29 (2003), 175-209.

10. J.-Y. Lee and R. V. Moody, Lattice substitution systems and model sets, Discrete Comput. Geom. 25 (2001), 173-201.

11. J.-Y. Lee, R. V. Moody, and B. Solomyak, Pure point dynamical and diffraction spectra, Ann. Henri Poincaré 3 (2002), 1003-1018.

12. R. V. Moody, Model sets: a survey, in From Quasicrystals to More Complex Systems, F. Axel and J.-P. Gazeau, eds., Les Editions de Physique, Springer-Verlag, Berlin, 2000, pp. 145-166.

13. B. Praggastis, Numeration systems and Markov partitions from self similar tilings, Trans. Amer. Math. Soc. 351(8) (1999), 3315-3349.

14. C. Radin and M. Wolff, Space tilings and local isomorphism, Geom. Dedicata 42 (1992), 355-360.

15. R. M. Robinson, Undecidability and nonperiodicity for tilings of the plane, Invent. Math. 12 (1971), 177-209.

16. M. Schlottmann, Generalized model sets and dynamical systems, in Directions in Mathematical Quasicrystals, M. Baake and R. V. Moody, eds., CRM Monograph Series, Vol. 13, AMS, Providence, RI, 2000, pp. 143-159.

17. B. Solomyak, Dynamics of self-similar tilings, Ergodic Theory Dynamical Systems 17 (1997), 695-738.

18. B. Solomyak, Corrections to "Dynamics of self-similar tilings," Ergodic Theory Dynamical Systems 19 (1999), 1685.

19. A. Vince, Digit tiling of Euclidean space, in Directions in Mathematical Quasicrystals, M. Baake and R. V. Moody, eds., CRM Monograph Series, Vol. 13, AMS, Providence, RI, 2000, pp. 329-370.

Received March 21, 2002, and in revised form December 12, 2002. Online publication April 22, 2003. 